\title{
PRECISION ELECTROWEAK PHYSICS AT LEP
}

\author{
Alain Blondel* \\ L.P.N.H.E. Ecole Polytechnique \\ 91128 Palaiseau, France ${ }^{\dagger}$
}

\begin{abstract}
Progress in precision measurements of electroweak observables at LEP continues steadily. The highlights of 1993 are: a new scan of the Z line shape with precise beam energy determination by resonant depolarization, leading to a measurement of the $\mathrm{Z}$ width with $1.5 \times 10^{-3}$ precision; first results on absolute cross sections with high-precision luminosity monitors; high efficiency $b$-tagging with microvertex detectors leading to $1 \%$ measurements of the $\mathrm{Z} \rightarrow b \bar{b}$ partial width, and of the forward-backward $b$ asymmetry with jet charge. All other electroweak measurements benefit from improved statistics. The basic symmetries of the Standard Model (SM) are verified, and electroweak radiative effects are now measured with a precision of a few $10^{-3}$. The results are compared with other precision electroweak measurements from SLC, neutrino scattering, and the $p \bar{p}$ colliders. The consequences of the recent evidence for the top quark are drawn out. Precision data begin to set significant limits on the Higgs boson mass and on alternative extensions to the Standard Model.
\end{abstract}

\footnotetext{
*Invited talks given at the Zeuthen Workshop on Physics at LEP200 and Beyond (Berlin, 1994), Tennessee International Workshop on Radiative Corrections (Gatlinburg, 1994) and SLAC Summer Institute (Stanford, 1994).

${ }^{\dagger}$ Mailing Address: CERN, PPE Division, CH-1211, Geneva 23, Switzerland.
}

(C)A. Blondel 1994 


\section{Introduction}

LEP performance has improved steadily since 1989 . The typical integrated luminosity recorded by the experiments has been: $1.2 \mathrm{pb}^{-1}$ in $1989,7.0 \mathrm{pb}^{-1}$ in 1990 , $13.0 \mathrm{pb}^{-1}$ in $1991,23.0 \mathrm{pb}^{-1}$ in 1992 , and $35.0 \mathrm{pb}^{-1}$ in 1993 . It is hoped to reach $60.0 \mathrm{pb}^{-1}$ in 1994 . The luminosity improvement is a product of several factors: (i) overall efficiency, now as high as 60\%; (ii) increase in 1992 of the number of bunches per beam from four to eight with the "Pretzel" scheme; (iii) better alignment, monitoring, and tuning procedures allowing now a high beam-beam tune shift of 0.04 ; and (iv) the luminosity lifetime, now well in excess of ten hours. Present records $(01 / 08 / 94)$ are a peak luminosity of $2.2 \times 10^{31} / \mathrm{cm}^{2} / \mathrm{s}$ (above design!) and integrated daily luminosity of $1.0 \mathrm{pb}^{-1} /$ day. Finally, the precise determination of the beam energy by resonant depolarization is essential for accurate measurement of the $\mathrm{Z}$ mass and width. These improvements are discussed in detail below; their effect on the precision to which the $Z$ line shape is now known is illustrated by Fig. 1.

Data were recorded by the four LEP experiments with efficiencies around $90 \%$ at center-of-mass energies around the $\mathrm{Z}$ pole. Scanning of the $\mathrm{Z}$ line shape took place in 1989, '90, '91, and '93; data were taken only at the peak of the resonance in 1992 and '94. No data has been recorded above $95 \mathrm{GeV}$ center-of-mass energy. With the cross sections decreasing very quickly, a very large step in energy is required to make this operation worthwhile. This should happen sometime in 1996, when the W-pair threshold is in reach with the addition of a large number (over 200) of superconducting RF cavities.

The results given here are based on the 1989-1993 data presented at the 1994 Glasgow conference, a total of eight million visible $Z$ decays, see Table 1 . They include preliminary numbers.

\section{A Synopsis of the Measured Quantities}

The building blocks of electroweak physics at the $\mathrm{Z}$ are measured cross sections for various final states, forward-backward and polarization asymmetries. Assuming that $\mathrm{Z}$ and photon exchange are the only processes that occur, they can all be expressed in terms of the chiral couplings, or more commonly, the vector and 


\begin{tabular}{|c|c|c|c|c|c|}
\hline & ALEPH & DELPHI & L3 & OPAL & LEP \\
\hline $\bar{q} q: \quad ' 90-' 91$ & 451 & 356 & 423 & 454 & 1684 \\
\hline '92 & 680 & 697 & 677 & 733 & 2787 \\
\hline '93 prel. & 653 & 677 & 658 & 653 & 2641 \\
\hline Total & 1784 & 1730 & 1758 & 1840 & 7112 \\
\hline$\ell^{+} \ell^{-}: 90-91$ & 55 & 27 & 40 & 58 & 180 \\
\hline '92 & 82 & 69 & 58 & 88 & 297 \\
\hline '93 prel. & 79 & 71 & 62 & 81 & 293 \\
\hline Total & 216 & 167 & 160 & 227 & 770 \\
\hline
\end{tabular}

Table 1: LEP experiments statistics in units of $10^{3}$ events used for the analysis of the $Z$ line shape and lepton forward-backward asymmetries.

axial-vector couplings. In the SM:

$$
\begin{aligned}
& g_{V f}=\left(g_{L f}+g_{R f}\right)=I_{L f}^{3}-2 Q_{f} \sin ^{2} \theta_{\mathrm{w}} \\
& g_{A f}=\left(g_{L f}-g_{R f}\right)=I_{L f}^{3} .
\end{aligned}
$$

The $\mathrm{Z} \rightarrow f \bar{f}$ partial width is given by:

$$
\Gamma_{f}=\frac{\alpha}{6 \sin ^{2} \theta_{\mathrm{w}} \cos ^{2} \theta_{\mathrm{w}}} \mathrm{M}_{\mathrm{Z}}\left(g_{L f} \stackrel{2}{ }+g_{R f}^{2}\right) .
$$

The total width is the sum over all open channels. Within very good limits, only the fermions of the first three families, with the exception of the top quark, contribute to the cross section.

Around the $\mathrm{Z}$ pole, the photon exchange is only a correction to the $\mathrm{Z}$ channel, which dominates the cross section and can then be written as:

$$
\sigma_{f}=12 \pi(\hbar c)^{2} \frac{s \Gamma_{e} \Gamma_{f}}{\left(s-\mathrm{M}_{\mathrm{Z}}^{2}\right)^{2}+s^{2} \frac{\Gamma_{Z}^{2}}{\mathrm{M}_{\mathrm{Z}}^{2}}} .
$$

One can easily see that forward-backward asymmetries or polarization asymmetries are sensitive to the following asymmetry of couplings:

$$
\mathcal{A}_{f} \equiv \frac{g_{L f}^{2}-g_{R f}^{2}}{g_{L f}^{2}+g_{R f}^{2}}=\frac{2 g_{V f} g_{A f}}{g_{V f}^{2}+g_{A f}^{2}} .
$$


For unpolarized beams, the forward-backward asymmetry is:

$$
\mathrm{A}_{\mathrm{FB}}^{(f)} \simeq \frac{3}{4} \mathcal{A}_{e} \mathcal{A}_{f}
$$

For the tau lepton, the polarization of the final state fermion is measurable as a function of polar angle. For unpolarized beams:

$$
\mathcal{P}_{\tau}(\cos \theta) \simeq-\frac{\mathcal{A}_{\tau}+\frac{2 \cos \theta}{1+\cos ^{2} \theta} \mathcal{A}_{e}}{1+\frac{2 \cos \theta}{1+\cos ^{2} \theta} \mathcal{A}_{e} \mathcal{A}_{\tau}}
$$

from which one can derive both $\mathcal{A}_{e}$ and $\mathcal{A}_{\tau}$.

Interesting observables are obtainable if longitudinal beam polarization is available. For example, the left-right asymmetry of $\mathrm{Z}$ production ${ }^{1,2}$

$$
\mathrm{A}_{\mathrm{LR}}=\frac{\sigma_{L}-\sigma_{R}}{\sigma_{L}+\sigma_{R}} \simeq \mathcal{A}_{e}
$$

and the forward-backward polarized asymmetry, ${ }^{3}$

$$
\mathrm{A}_{\mathrm{FB}}^{\mathrm{pol}(f)}=\frac{\left(\sigma_{L, F}-\sigma_{R, F}\right)-\left(\sigma_{L, B}-\sigma_{R, B}\right)}{\left(\sigma_{L, F}+\sigma_{R, F}\right)+\left(\sigma_{L, B}+\sigma_{R, B}\right)} \simeq \frac{3}{4} \mathcal{A}_{f}
$$

The values of neutral current couplings and their sensitivity to $\sin ^{2} \theta_{\mathrm{w}}^{\text {eff }}$ are given in Table 2.

\begin{tabular}{|c|cc|cc|cc|}
\hline \hline$f$ & $I_{3 f}$ & $Q_{f}$ & $g_{A f}$ & $g_{V f}$ & $\mathcal{A}_{f}$ & $\frac{\partial \mathcal{A}_{f}}{\partial \sin ^{2} \theta_{\mathrm{w}}^{\mathrm{eff}}}$ \\
\hline$\nu$ & $1 / 2$ & 0 & $1 / 2$ & $1 / 2$ & 1 & 0 \\
$e$ & $-1 / 2$ & -1 & $-1 / 2$ & -0.04 & 0.16 & -7.9 \\
$u$ & $1 / 2$ & $2 / 3$ & $1 / 2$ & 0.19 & 0.69 & -3.5 \\
$d$ & $-1 / 2$ & $-1 / 3$ & $-1 / 2$ & -0.35 & 0.94 & -0.6 \\
\hline \hline
\end{tabular}

Table 2: Numerical values of quantum numbers, neutral current couplings, chiral coupling asymmetry $\mathcal{A}_{f}$, and sensitivity of $\mathcal{A}_{f}$ for the four types of fermions. The value of $\sin ^{2} \theta_{\mathrm{w}}^{\mathrm{eff}}$ is 0.23 .

\subsection{A Strategy of Tests and Radiative Effects}

One can organize the measurements at LEP in two broad classes: (i) the measurements providing tests of the $\mathrm{SU}(2)_{\mathrm{L}} \times \mathrm{U}(1)$ gauge structure, and (ii) the measurements which probe electroweak radiative effects. 
The main consequence of $\mathrm{SU}(2)_{\mathrm{L}} \times \mathrm{U}(1)$ invariance is Universality in a global sense: the couplings of particles with the same quantum numbers should be the same, independently of their family. This is best tested with leptons. Furthermore, the chiral couplings of the $\mathrm{Z}$ to fermions should all obey the formulae of Eq. (1). After correction for radiative effects, the same value of $\sin ^{2} \theta_{\mathrm{w}}$ should match all measured couplings.

Besides QED radiative effects (emission of real or virtual photons) which are conceptually straightforward, LEP observables are sensitive to electroweak (propagator or vertex) radiative effects. Electroweak corrections are sensitive ${ }^{4}$ to heavy, yet undiscovered particles, such as the top quark or the Higgs boson, in an inclusive way. There are four ${ }^{5-10}$ main radiative effects at the $\mathrm{Z}$ pole:

- The running of the QED coupling constant $\alpha\left(q^{2}\right)$ from $q^{2}=0$ to $q^{2}=\mathrm{M}_{\mathrm{Z}}^{2}$.

- The isospin-breaking loop corrections to the $\mathrm{W}$ and $\mathrm{Z}$ propagators. They are absorbed conveniently in the $\rho$ parameter, $\rho=1+\Delta \rho$.

- The running of the $\mathrm{Z}$ self-energy, absorbed in the parameter $\Delta_{3 Q}$.

- The $\mathrm{Z} \rightarrow b \bar{b}$ vertex correction.

One more parameter, $\Delta \mathrm{r}^{\mathrm{ew}}$, is necessary for the $\mathrm{W}$ mass. The propagator corrections modify Eq. (1) by an overall scaling factor $\sqrt{\rho}$ and a global change of $\sin ^{2} \theta_{\mathrm{w}}$ in a universal way. Nonuniversal corrections are small and-with the notable exception of the $\mathrm{Z} \rightarrow b \bar{b}$ vertex - insensitive to heavy physics. Furthermore, all asymmetries with unpolarized beams and the most precise asymmetry with polarized beams are proportional to the electron coupling $\mathcal{A}_{e}$, while the sensitivity to $\sin ^{2} \theta_{\mathrm{w}}$ of hadronic asymmetries is contained in the $\mathcal{A}_{e}$ term [see Eq. (5) and Table 2]. It is, therefore, convenient to express all asymmetry measurements at LEP in terms of the effective weak mixing angle ${ }^{11}$ defined as:

$$
\sin ^{2} \theta_{\mathrm{w}}^{\mathrm{eff}} \equiv \frac{1}{4}\left(1-\frac{g_{V e}}{g_{A e}}\right)
$$

where the ratio $\frac{g_{V e}}{g_{A e}}$ is extracted from pole asymmetries. This definition absorbs vertex corrections for leptons but not for quarks. See Refs. 6, 7, and 12 for various avatars of the concept. This definition of $\sin ^{2} \theta_{\mathrm{w}}^{\mathrm{eff}}$ and the $\overline{\mathrm{MS}}$ one ${ }^{13}$ are very close. ${ }^{10,14}$

The relations between LEP observables, the Fermi constant $\mathrm{G}_{\mathrm{F}}$, and the QED running constant $\alpha\left(\mathrm{M}_{\mathrm{Z}}^{2}\right)^{-1}=128.87 \pm 0.12$ (Ref. 15) can be written in terms of 


\begin{tabular}{|c|c|c|c|}
\hline Quantity & Main Technologies & Physics Outputs & $\begin{array}{l}\text { Relative } \\
\text { Precision }\end{array}$ \\
\hline \multicolumn{4}{|l|}{ Line shape } \\
\hline $\mathrm{M}_{\mathrm{Z}}$ & $\begin{array}{l}\text { Absolute energy scale } \\
\text { relative cross sections } \\
\text { line-shape fit (QED rad. corr.) }\end{array}$ & input & $5 \times 10^{-5}$ \\
\hline$\Gamma_{\mathrm{Z}}$ & $\begin{array}{l}\text { Relative energy scale } \\
\text { relative cross sections } \\
\text { line-shape fit (QED rad. corr.) }\end{array}$ & $\Delta \rho$ & $1.5 \times 10^{-3}$ \\
\hline$\sigma_{\text {had }}^{\text {peak }, 0}$ & Absolute cross sections & $\begin{array}{c}\mathrm{N}_{\nu} \cdot \frac{\Gamma_{\text {inv }}}{\Gamma_{\ell}} \\
\text { test } \mathrm{SU}(2)_{\mathrm{L}} \times \mathrm{U}(1)\end{array}$ & $3 \times 10^{-3}$ \\
\hline $\mathrm{R}_{\ell} \equiv \frac{\Gamma_{\mathrm{had}}}{\Gamma_{\ell}}$ & lepton, hadron event selection & $\begin{array}{l}\text { test universality } \\
f\left(\alpha_{s}, \sin ^{2} \theta_{\mathrm{w}}^{\mathrm{eff}}, \delta_{v b}\right)\end{array}$ & $\begin{array}{l}4 \times 10^{-3} \\
2 \times 10^{-3}\end{array}$ \\
\hline $\mathrm{R}_{b} \equiv \frac{\Gamma_{b}}{\Gamma_{\mathrm{had}}}$ & $b$-tagging & $\delta_{v b}$ & $10^{-2}$ \\
\hline Asymmetries & & $\sin ^{2} \theta_{\mathrm{w}}^{\mathrm{eff}}$ & $2 \times 10^{-3}$ \\
\hline
\end{tabular}

Table 3: Synopsis of precision neutral current observables at the $Z$ pole. 
these universal electroweak corrections $\Delta \rho, \Delta_{3 Q}, \Delta \mathrm{r}^{\mathrm{ew}}$, and $\delta_{v b}$ as $: 9,10,16,17$

$$
\begin{aligned}
\mathrm{M}_{\mathrm{Z}}^{2} & =\frac{\pi \alpha\left(\mathrm{M}_{\mathrm{Z}}^{2}\right)}{\sqrt{2} \mathrm{G}_{\mathrm{F}}(1+\Delta \rho)\left(1+\Delta_{3 Q}\right) \sin ^{2} \theta_{\mathrm{w}}^{\mathrm{eff}} \cos ^{2} \theta_{\mathrm{w}}^{\mathrm{eff}}} ; \\
\Gamma_{\ell} & =\frac{\mathrm{G}_{\mathrm{F}} \mathrm{M}_{\mathrm{Z}}^{3}}{24 \sqrt{2} \pi}[1+\Delta \rho]\left[1+\left(\frac{g_{V \ell}}{g_{A \ell}}\right)^{2}\right]\left(1+\frac{3}{4} \frac{\alpha}{\pi}\right) \\
\Gamma_{b} & =\Gamma_{d}\left(1+\delta_{v b}\right) ; \\
\mathrm{M}_{\mathrm{W}}^{2} & =\frac{\pi \alpha\left(\mathrm{M}_{\mathrm{Z}}^{2}\right)}{\sqrt{2} \mathrm{G}_{\mathrm{F}}\left(1-\Delta \mathrm{r}^{\mathrm{ew}}\right)\left(1-\frac{\mathrm{M}_{\mathrm{W}}^{2}}{\mathrm{M}_{\mathrm{Z}}^{2}}\right)}
\end{aligned}
$$

Table 3 summarizes the main observables, their physics output, and the most critical technique involved. With this specific choice, these observables are almost uncorrelated, from both points of view of statistical and systematic errors.

\section{The Z Line Shape}

The measurements of cross sections by the four LEP detectors are reported in Refs. 18-20, and 22.

\subsection{Luminosity Measurement}

The determination of luminosity is based on counting low angle Bhabha events $e^{+} e^{-} \rightarrow e^{+} e^{-}$. Luminosity monitors consist of electromagnetic calorimeters, with good spatial resolution, positioned very accurately on each side of the experiments near the beam pipe. Bhabha events appear as two back-to-back electromagnetic showers, each carrying the full beam energy, as shown in Fig. 2. A thorough discussion of the luminosity measurement can be found in the line shape publications by the experiments, and in Refs. 23-26. For a minimum angle $\theta_{\min }$ of $29 \mathrm{mrad}$, the selected cross section exceeds $100 \mathrm{nb}$. The statistical accuracy is better than $10^{-3}$. The main experimental challenge comes from the $1 / \theta_{\min }^{2}$ dependence of the selected Bhabha cross section. An uncertainty on the inner radius $R$ of the sensitive region of the luminosity calorimeter induces an error on the measured cross section:

$$
\frac{\Delta \sigma_{\text {Bhabha }}}{\sigma_{\text {Bhabha }}}=2 \times \frac{\Delta \theta_{\text {min }}}{\theta_{\text {min }}} \simeq 2 \times \frac{\Delta R}{R} .
$$



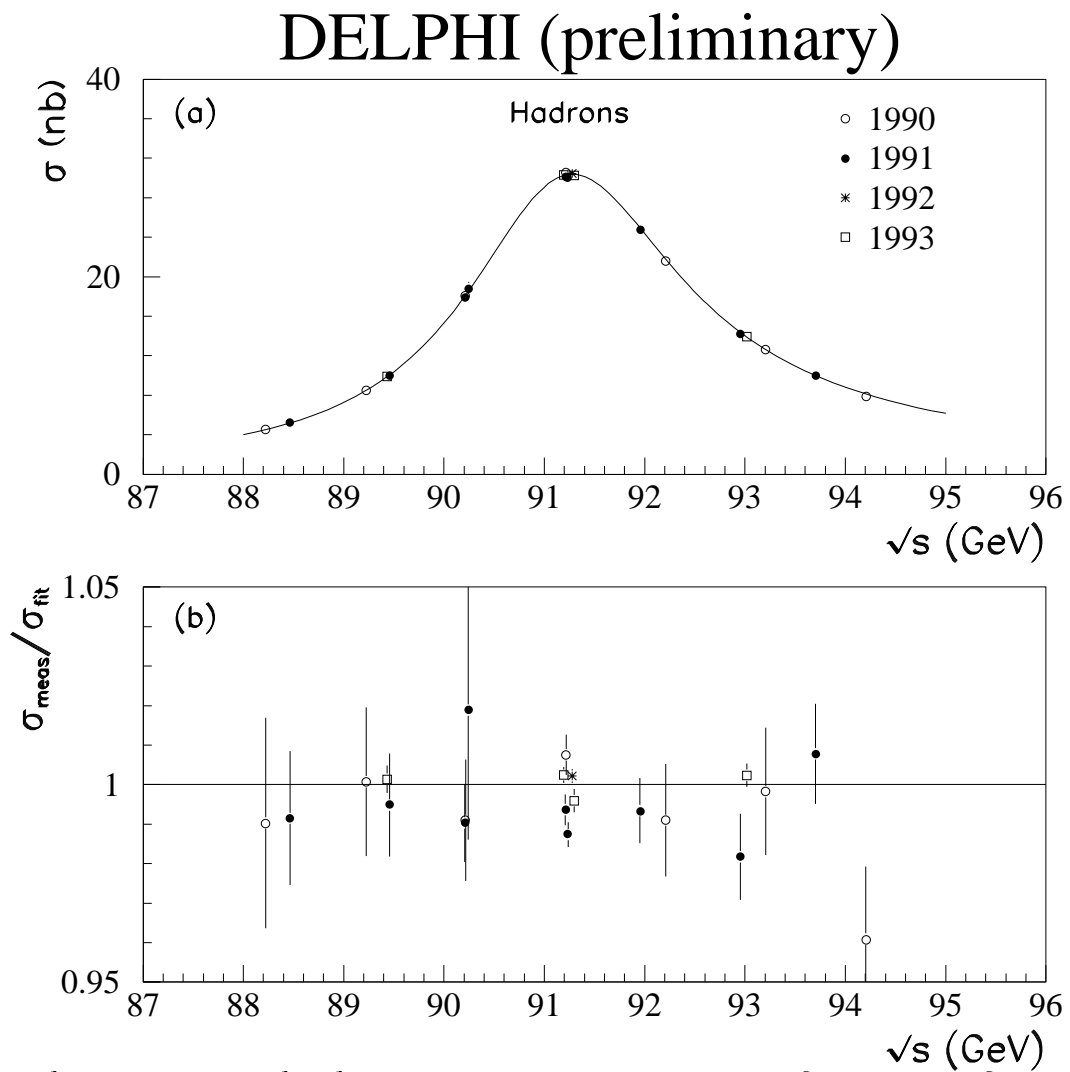

Figure 1: The DELPHI hadronic cross sections as a function of center-of-mass energy. The square points show the considerable statistical improvement from the 1993 scan. 
Figure 2: A luminosity event in the ALEPH SiCAL luminosity monitor. Left: front view $(x-y)$ : The showers of the electron (side A) and positron (side B) are displayed on the same graph. The silicon pads are represented with dots of size proportional to the energy collected. The ring shows the radial pad row at which the clusters are reconstructed. Right: Side view (r-z) of the two showers, with the corresponding energy profiles. 


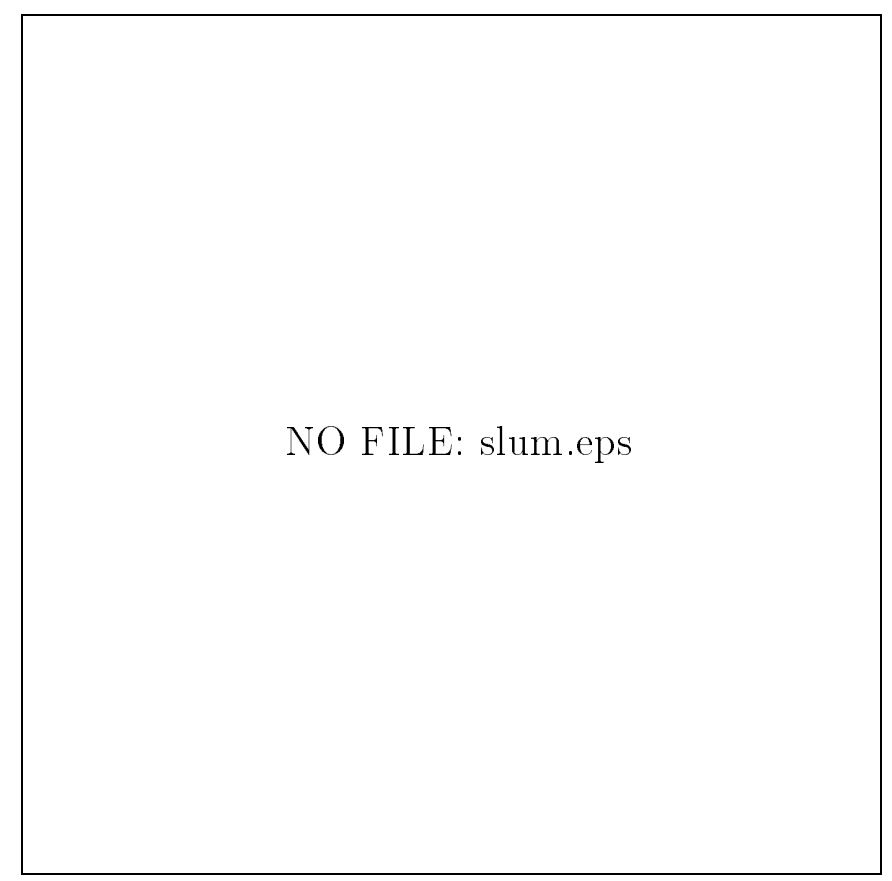

Figure 3: Side view of the L3 detector, showing the new low-angle silicon luminosity tracker (SLUM). 
For the typical value of $\mathrm{R}(5 \mathrm{~cm})$, a precision well under $25 \mu \mathrm{m}$ is required to match the statistical accuracy. The LEP experiments have upgraded their luminosity monitors to provide high-precision knowledge of the inner edge. The ALEPH silicon calorimeter (SiCAL) ${ }^{24}$ was operational from the middle of the 1992 data onwards. A SiCAL event is shown in Fig. 2. SiCAL is a silicon-tungsten sandwich with precision-machined planes of silicon pads for energy readout. The fiducial cut $\theta_{\min }$ is made on pad boundaries, where the position resolution is optimum. The radius of the pad boundaries is known with a precision of better than ten microns.

The other experiments have made similar improvements to their luminosity measurement: OPAL ${ }^{25}$ with a detector similar to the ALEPH SiCAL, operational in 1993, giving a precision of $0.07 \%$; L3 (Ref. 26) with a precision silicon tracker positioned in front of the BGO luminosity calorimeter, Fig. 3, operational in 1993, obtains a $0.16 \%$ precision; DELPHI with a silicon telescope operational from 1992, and a lead/scintillator/silicon sandwich that should be operational in 1994. The breakdown of experimental systematic errors for ALEPH, OPAL, and L3 is shown in Table 4.

At low angles, the $\mathrm{Z}$ contribution is less than $5 \cdot 10^{-4}$. The $\mathrm{Z}-\gamma$ interference is small at the $Z$ pole but can be as large as $6 \cdot 10^{-3}$ off the pole, leading to a small correction that affects only the $\mathrm{Z}$ mass. Other low-angle QED processes such as $e^{+} e^{-} \rightarrow \gamma \gamma$ are small $\left(2 \times 10^{-4}\right)$ and well calculable. The calculation of radiative corrections to the Bhabha scattering cross section itself is made delicate by the interplay of experimental cuts with higher order processes that are not simulated. No single-event generator has a complete account of the corrections, so the estimate presently involves a combination of: (i) event generation with $\mathrm{BH}-$ LUMI ${ }^{27}$ a multiphoton $\mathcal{O}(\alpha)$ Monte Carlo with exclusive exponentiation (many radiative photons are generated, assuming successive occurrence of the first-order process); (ii) complete electroweak first-order QED calculations $;^{28}$ and (iii) estimate of higher order processes by leading-log and second-order calculations. ${ }^{29,30}$ The present estimate of the theoretical error is $\pm 0.25 \%$ for a minimum angle of $25 \mathrm{mrad}$, larger than the experimental one. Hard work is taking place to reduce the error ${ }^{31}$ down to $<10^{-3}$. 


\begin{tabular}{|l|r|r|r|}
\hline & \multicolumn{3}{|c|}{ Syst. Err. (10-4) } \\
Source & ALEPH & OPAL & L3 \\
\hline Backgrounds & & & \\
- Beam particles & 0.3 & 0.1 & - \\
- "Physics" sources & 1. & 0.1 & - \\
Trigger eff. & 0.02 & $<0.01$ & - \\
Reconstruction & 0.1 & - & - \\
Radial fid. cuts: & & & \\
- mech. precision & 2.9 & 3.6 & 3.3 \\
- beam position & 3.0 & 2.1 & - \\
- long. position & 3.5 & 0.6 & 6.0 \\
- asymmetry cuts & 2.6 & 2.6 & - \\
Shower param. and & & & \\
energy cuts & 3.6 & 3.8 & - \\
Acoplanarity cut & 0.5 & - & - \\
or evt. selection & - & - & 9.0 \\
Simulation stat. & 6.0 & 3.7 & 10. \\
\hline TOTAL Exp. Error & 9.5 & 7.2 & 15.6 \\
\hline
\end{tabular}

Table 4: Systematic errors for the ALEPH SiCAL, OPAL Si-W, and L3 Sitracker+BGO luminosity measurement.

\subsection{Selection of Hadronic and Leptonic Events}

Decays of the $\mathrm{Z}$ into $q \bar{q}$ pairs are not separately identified but generically labeled as hadrons. The selection efficiency is very large, typically $97 \%$ to $99 \%$, and the resulting systematic errors rather small, see Table 5. Energy-dependent corrections come from the subtraction of the "two-photon" background and from energy variation of the selection efficiency, leading to systematic errors of less than $1 \mathrm{MeV}$ on the $\mathrm{Z}$ width. 


\begin{tabular}{|l||c|c|c|c|c|c|c|c|}
\hline \multicolumn{1}{|c||}{} & \multicolumn{2}{c|}{ ALEPH } & \multicolumn{2}{c|}{ DELPHI } & \multicolumn{2}{|c|}{ L3 } & \multicolumn{2}{c|}{ OPAL } \\
\cline { 2 - 9 } & $' 92$ & $\begin{array}{c}' 93 \\
\text { prel. }\end{array}$ & 92 & $\begin{array}{c}' 93 \\
\text { prel. }\end{array}$ & ${ }^{\prime} 92$ & $\begin{array}{c}93 \\
\text { prel. }\end{array}$ & & $\begin{array}{c}' 93 \\
\text { prel. }\end{array}$ \\
\hline \hline $\mathcal{L}^{\text {exp. }}$ & $0.15 \%$ & $0.09 \%$ & $0.38 \%$ & $0.28 \%$ & $0.5 \%$ & $0.16 \%$ & $0.41 \%$ & $0.07 \%$ \\
\hline$\sigma_{\text {had }}$ & $0.14 \%$ & $0.14 \%$ & $0.13 \%$ & $0.13 \%$ & $0.15 \%$ & $0.11-0.14 \%$ & $0.20 \%$ & $0.20 \%$ \\
$\sigma_{\mathrm{e}}$ & $0.4 \%$ & $0.4 \%$ & $0.59 \%$ & $1.2 \%$ & $0.3 \%$ & $0.25-0.76 \%$ & $0.22 \%$ & $0.23 \%$ \\
$\sigma_{\mu}$ & $0.5 \%$ & $0.5 \%$ & $0.37 \%$ & $0.5 \%$ & $0.5 \%$ & $0.45-0.57 \%$ & $0.19 \%$ & $0.22 \%$ \\
$\sigma_{\tau}$ & $0.3 \%$ & $0.3 \%$ & $0.63 \%$ & $0.8 \%$ & $0.7 \%$ & $0.54 \%$ & $0.44 \%$ & $0.46 \%$ \\
\hline $\mathrm{A}_{\mathrm{FB}}^{(e)}$ & 0.0029 & 0.0029 & 0.003 & 0.003 & 0.002 & 0.005 & 0.002 & 0.002 \\
$\mathrm{~A}_{\mathrm{FB}}^{(\mu)}$ & 0.001 & 0.001 & 0.001 & 0.002 & 0.002 & 0.001 & 0.001 & 0.001 \\
$\mathrm{~A}_{\mathrm{FB}}^{(\tau)}$ & 0.0005 & 0.0005 & 0.0017 & 0.002 & 0.003 & 0.004 & 0.002 & 0.002 \\
\hline
\end{tabular}

Table 5: The experimental systematic errors for the analysis of the $Z$ line shape and lepton forward-backward asymmetries. The errors quoted do not include the common uncertainties due to the LEP energy calibration and to the theoretical error on the Bhabha cross-section calculation. For L 3 cross sections, the range of errors corresponds to the different center-of-mass energies. For the treatment of correlations between the errors for different years, see Refs. 18-20, 22.

Leptonic decays of the $\mathrm{Z}, e^{+} e^{-} \rightarrow e^{+} e^{-}, e^{+} e^{-} \rightarrow \mu^{+} \mu^{-}$, and $e^{+} e^{-} \rightarrow \tau^{+} \tau^{-}$offer much simpler topologies than hadronic decays. They are however less frequent $(1: 20)$, and, since they have fewer tracks, are easier to miss. The experimental uncertainties are summarized in Table 5 .

The $e^{+} e^{-}$mode is affected by the t-channel Bhabha scattering process, which has to be subtracted. Leading second-order calculations are available, ${ }^{32}$ and the procedure introduces a negligible systematic error.

\subsection{The Beam Energy}

The LEP data were taken in 1990-1991 at seven different center-of-mass energies interspaced by $1 \mathrm{GeV}$ from $88.25 \mathrm{GeV}$ to $94.25 \mathrm{GeV}$. In 1992 , all data were taken at the $\mathrm{Z}$ pole. In 1993 , a scan of the $\mathrm{Z}$ line shape was performed again at the energies of 89.4, 91.2, and $93 \mathrm{GeV}$. The 1993 energies were chosen (i) to minimize the statistical error on $\Gamma_{Z}$, and (ii) to obtain beam polarization that allows precise 
energy calibration by resonant depolarization for all three points. ${ }^{33}$

Transverse spin polarization builds up in a storage ring by the Sokolov-Ternov effect. ${ }^{34}$ It has been observed in all storage rings where it has been searched for, ${ }^{35}$ and in LEP since 1990 (Ref. 36). Resonant depolarization has been used previously in $e^{+} e^{-}$machines, providing accurate measurements of the masses of the $\mathrm{J} / \psi, \psi^{\prime}, \Upsilon, \Upsilon^{\prime}$, at VEPP4 in Novosibirsk, ${ }^{37,38}$ at DORIS in Hamburg, ${ }^{39}$ and at CESR in Cornell. ${ }^{40}$ It was first performed in LEP in 1991 (Ref. 41).

The spin precession frequency is determined as follows: a fast kicker generates a periodic perturbation to the beam (and its spin). If the perturbation is in resonance with the spin precession, one observes a sharp decrease or even reversal of the measured polarization. The number of spin precessions per turn, or spin tune, $\nu$ is obtained by dividing the spin precession frequency by the revolution frequency. It is directly related to the beam energy by the anomalous magnetic moment $a_{e}=\frac{g_{e}-2}{2}=1.1596521884(43) \times 10^{-3}$ and the mass $m_{e}=0.51099906(15) \mathrm{MeV}$ of the electron:

$$
\nu=a_{e} \gamma=\frac{g_{e}-2}{2} \frac{\mathrm{E}_{\text {beam }}}{m_{e}}=\frac{\mathrm{E}_{\text {beam }}(\mathrm{GeV})}{0.4406486(1)} \simeq 103.5 \text { at the } \mathrm{Z} \text { pole. }
$$

The intrinsic resolution of the method is better than $200 \mathrm{KeV},{ }^{42}$ see Fig. 4. However, energy measurements are delicate and performed only seldomly, four times in 1991. In 1993, they were made more compatible with normal physics operation and performed 25 times, roughly in a third of physics fills. The extrapolation to the whole scan data requires tracing in time the properties of the magnets, current, field, and temperature, as well as the geometrical properties of the ring. The analysis of the accumulated data is performed in collaboration by the accelerator physicists and members of the LEP collaborations within the LEP Energy working group. ${ }^{43}$

The most spectacular source of energy variations comes from ground motion. Because of the strong focusing of LEP, these movements are amplified by a factor of nearly $10^{4}$, so that a small expansion by $\pm 10^{-8}$ leads to a potential error on the $\mathrm{Z}$ mass and width of $10 \mathrm{MeV}$. Terrestrial tides ${ }^{44}$ are one strong cause of such variations and were indeed observed, ${ }^{45}$ see Fig. 5. All known sources of fluctuations being removed, the LEP energy calibrations of 1993 still show a full swing of more than $20 \mathrm{MeV}$ of the beam energy. Careful investigations of the beam orbit measurements ${ }^{46,47}$ show that the observed energy jumps are correlated 


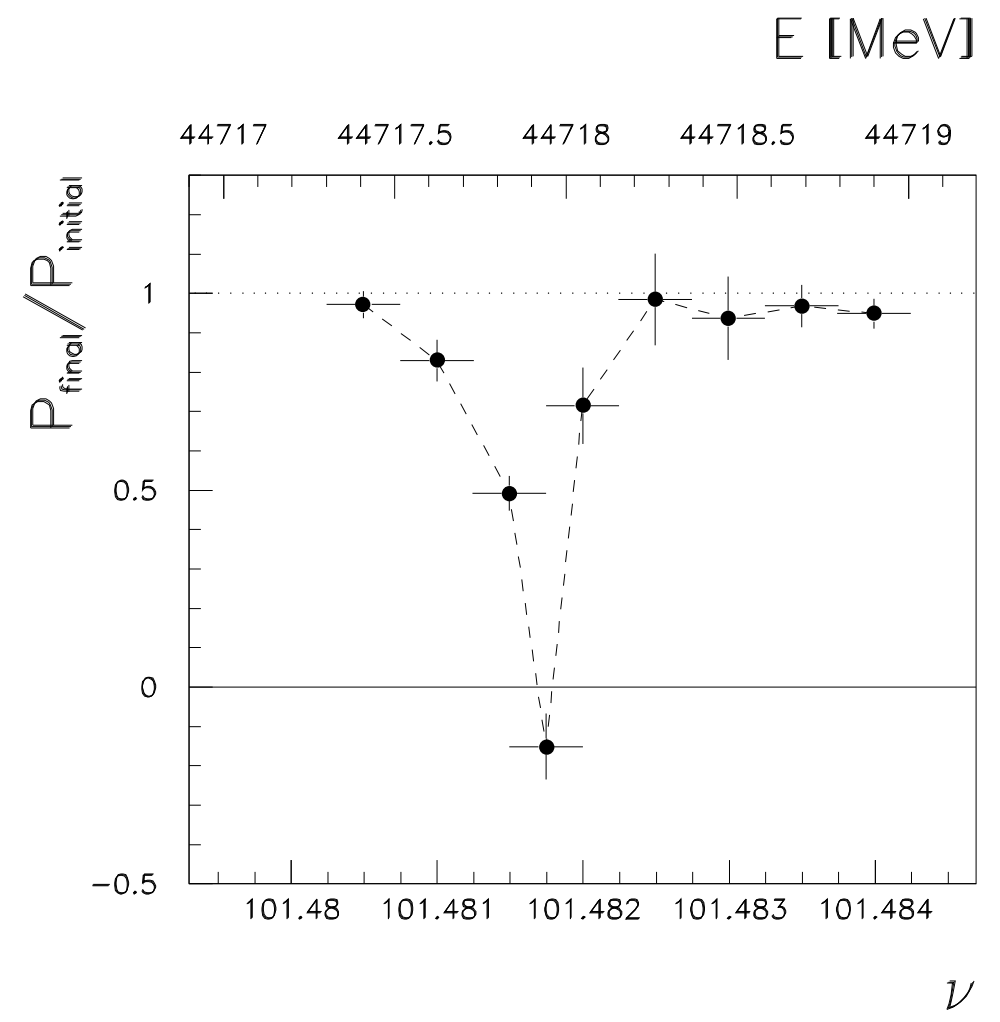

Figure 4: Measurement of the width of the artificial depolarizing resonance, showing a width of $200 \mathrm{KeV}$. 
Figure 5: Beam-energy variations measured over 24 hours compared to the expectation from the tides. 
with orbit movement, especially visible in September 1993 when a century record of rainfall took place!

The present knowledge of the beam energy leads to systematic errors of $4 \mathrm{MeV}$ on the $\mathrm{Z}$ mass, and $3 \mathrm{MeV}$ on the $\mathrm{Z}$ width, before correction with the orbit. It is hoped to reduce these errors to about $2 \mathrm{MeV}$ when the analysis of the measured orbits is complete.

\subsection{Forward-Backward Asymmetries for Leptons}

The lepton forward-backward asymmetry $\mathrm{A}_{\mathrm{FB}}^{(\ell)}$ is a steep function of center-of-mass energy, as can be seen in Fig. 6. This leads to some sensitivity to initial state radiation and beam energy uncertainties, which induces a correlation with the $\mathrm{Z}$ mass. The lepton forward-backward asymmetry can also be used to constrain the Z-photon interference term. For these reasons, the line-shape fit includes the leptonic forward-backward asymmetries.

The initial state radiation effect is treated with great detail in Ref. 49 and implemented in fitting formulae, such as MIZA $^{51}$ and ZFITTER, ${ }^{52}$ together with photon-exchange terms. It is believed that the QED corrected Z-pole asymmetry of Eq. (5) can be extracted from the measured one with an accuracy of 0.0008 (Ref. 49). 


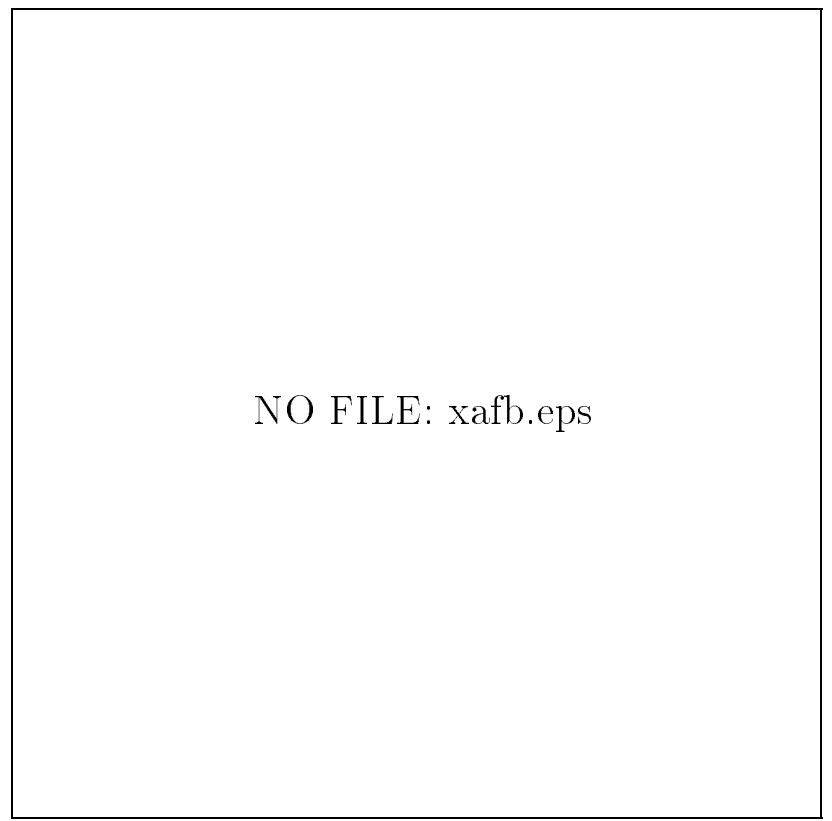

Figure 6: The OPAL fitted forward-backward asymmetries for electron, muon, tau, and inclusive lepton final states. The lines are the results of a global fit to the line shape and lepton asymmetries. 
Figure 7: One standard deviation contours (39\% probability) in the $\mathrm{R}_{\ell}-\mathrm{A}_{\mathrm{FB}}^{(\ell)}$ plane. The SM prediction for $\mathrm{M}_{\mathrm{Z}}=91.1895 \mathrm{GeV}, \mathrm{M}_{\mathrm{t}}=150 \mathrm{GeV}, \mathrm{M}_{\mathrm{H}}=300 \mathrm{GeV}$, $\alpha_{s}=0.123$ is shown as a dot. The arrows correspond to SM predictions for $50<\mathrm{M}_{\mathrm{t}}(\mathrm{GeV})<250,60<\mathrm{M}_{\mathrm{H}}(\mathrm{GeV})<1000$, and $\alpha_{s}\left(\mathrm{M}_{\mathrm{Z}}^{2}\right)=0.123 \pm 0.006$. The arrows point towards increasing $\mathrm{M}_{\mathrm{t}}, \mathrm{M}_{\mathrm{H}}$, and $\alpha_{s}$. 
The extracted asymmetry is insensitive to the absolute energy scale of LEP, but it is sensitive to the relative distance of the scan points from the $\mathrm{Z}$ peak. Therefore, a $10 \mathrm{MeV}$ point-to-point error results in an uncertainty of 0.0008 on $\mathrm{A}_{\mathrm{FB}}^{(\ell)}$, fully correlated for the three lepton types. Because of the interference with the t-channel, the dependence of the $e^{+} e^{-}$asymmetry on beam energy is of opposite sign than for the other two leptons, see Fig. 6. The effect is thus reduced in the average lepton asymmetry.

\begin{tabular}{|l|cccc|cc||}
\hline & ALEPH & DELPHI & L3 & OPAL & Average & $\chi^{2} /$ d.o.f.
\end{tabular}

Table 6: Lepton universality tests, $\mathrm{R}_{\ell}$ and $\mathrm{A}_{\mathrm{FB}}^{(\ell)}$, are extracted from the nineparameter fits to the data of the four LEP experiments.

\begin{tabular}{|l|cccc|cc||}
\hline & ALEPH & DELPHI & L3 & OPAL & Average & $\chi^{2} /$ d.o.f. \\
\hline \hline $\mathrm{M}_{\mathrm{Z}}(\mathrm{MeV})$ & $91191.5 \pm 3.3$ & $91186.9 \pm 3.3$ & $91190.0 \pm 3.6$ & $91186.2 \pm 3.6$ & $91188.7 \pm 1.7 \pm 4$ & $1.6 / 3$ \\
$\Gamma_{\mathrm{Z}}(\mathrm{MeV})$ & $2495.9 \pm 5.5$ & $2495.1 \pm 5.2$ & $2504.0 \pm 5.1$ & $2494.6 \pm 5.5$ & $2497.6 \pm 2.7 \pm 2.7$ & $2.2 / 3$ \\
$\sigma_{\text {had }}^{\text {peak }, 0}(\mathrm{nb})$ & $41.59 \pm 0.08$ & $41.26 \pm 0.13$ & $41.45 \pm 0.11$ & $41.48 \pm 0.12$ & $41.49 \pm 0.05 \pm 0.10$ & $4.7 / 3$ \\
$\mathrm{R}_{\ell}$ & $20.730 \pm 0.078$ & $20.690 \pm 0.090$ & $20.859 \pm 0.088$ & $20.864 \pm 0.076$ & $20.795 \pm 0.040$ & $3.4 / 3$ \\
$\mathrm{~A}_{\mathrm{FB}}^{(\ell)} \times 10^{4}$ & $216 \pm 25$ & $160 \pm 28$ & $168 \pm 35$ & $137 \pm 24$ & $170 \pm 14 \pm 8$ & $5.5 / 3$
\end{tabular}

Table 7: Line-shape parameters from the four LEP experiments, ${ }^{18-20,22}$ combined according to Ref. 48. 


\subsection{Results on the Z Line Shape}

Once the cross sections, asymmetries, and energies are determined, a fit is performed to unfold the pure $\mathrm{Z}$ contribution from the photon contribution and the initial state radiation.

Two different fits are usually performed. First, to verify lepton universality, a nine-parameter fit: $\mathrm{M}_{\mathrm{Z}}, \Gamma_{\mathrm{Z}}, \sigma_{\text {had }}^{\text {peak }, 0}, \mathrm{R}_{e}, \mathrm{R}_{\mu}, \mathrm{R}_{\tau}, \mathrm{A}_{\mathrm{FB}}^{(e)}, \mathrm{A}_{\mathrm{FB}}^{(\mu)}$, and $\mathrm{A}_{\mathrm{FB}}^{(\tau)}$. The results are shown in Table 6 and Fig. 7. The accuracy of the test is $0.35 \%$. Within this precision, the values of $\mathrm{R}_{\ell}$ and $\mathrm{A}_{\mathrm{FB}}^{(\ell)}$ for all leptons are consistent with each other, with a maximum discrepancy of two standard deviations for $\mathrm{A}_{\mathrm{FB}}^{(\tau)}$. This being in agreement with lepton universality, one can thus make this assumption and fit for one leptonic width $\Gamma_{\ell}$ defined as the partial $\mathrm{Z}$ decay width into a pair of massless leptons, and one asymmetry $\mathrm{A}_{\mathrm{FB}}^{(\ell)}$. The result is shown in Table 7 .

The correlations between these parameters, given in Table 8, are small. The LEP averages are performed taking into account the common systematic errors: (i) the beam energy errors; (ii) a common error of $0.25 \%$ on absolute cross sections from the theoretical uncertainty on $\sigma_{\text {Bhabha }}$; and (iii) a common uncertainty of 0.0008 on lepton asymmetries due to the accuracy of the QED radiation. The agreement between experiments is acceptable, as shown by the values of $\chi^{2}$ for three degrees of freedom given in Table 7 .

One can extract from these numbers the values of $\mathrm{N}_{\nu}$, so that the line-shape results for LEP can be summarized as:

$$
\begin{aligned}
\mathrm{M}_{\mathrm{Z}} & =91.1895 \pm 0.0017 \pm 0.0040_{\mathrm{LEP}} \\
\Gamma_{\mathrm{Z}} & =2.4969 \pm 0.0027 \pm 0.0027_{\mathrm{LEP}} \\
\mathrm{N}_{\nu} & =2.988 \pm 0.010 \pm 0.019_{\mathrm{th}} \\
\mathrm{R}_{\ell} & =20.795 \pm 0.040
\end{aligned}
$$

This, clearly, is consistent with three species of light neutrinos (with mass smaller than $\mathrm{M}_{\mathrm{Z}} / 2$ ). An important derived parameter is the leptonic partial width:

$$
\Gamma_{\ell}=83.96 \pm 0.18 \mathrm{MeV} .
$$

From the average value of $\mathrm{A}_{\mathrm{FB}}^{(\ell)}$, one can derive a value of the effective weak mixing angle:

$$
\sin ^{2} \theta_{\mathrm{w}}^{\mathrm{eff}}=0.23107 \pm 0.00090
$$




\begin{tabular}{||c|ccc|c||}
\hline & $\Gamma_{\mathrm{Z}}$ & $\sigma_{\text {had }}^{\text {peak }, 0}$ & $\mathrm{R}_{\ell}$ & $\mathrm{A}_{\mathrm{FB}}^{(\ell)}$ \\
\hline $\mathrm{M}_{\mathrm{Z}}$ & 0.04 & 0.01 & -0.01 & 0.04 \\
$\Gamma_{\mathrm{Z}}$ & & -0.11 & 0.01 & 0.00 \\
$\sigma_{\text {had }}^{\text {peak }, 0}$ & & & 0.13 & 0.00 \\
$\mathrm{R}_{\ell}$ & & & & 0.01 \\
\hline
\end{tabular}

Table 8: Correlation matrix for the parameters of Table 7.

\section{Partial Widths into Specific Flavors}

Because it belongs to the same multiplet as the heavy top quark, the $\mathrm{Z} \rightarrow b \bar{b}$ partial width receives a specific vertex correction, sensitive uniquely to the top quark mass. ${ }^{53}$ In order to measure the $\mathrm{Z} \rightarrow b \bar{b}$ partial width or the $b$ forward-backward asymmetry, the first step is identification of $b$ events, or " $b$-tagging." $b$-tagging is interesting for many reasons. Besides allowing electroweak measurements, it is a key tool in selecting clean $b$ samples for study of exclusive $b$-quark properties, $B^{0} \overline{B^{0}}$ mixing, and even as a secondary tool for searching for the Higgs boson, which is expected to decay primarily into $b$-quarks, if it is not too heavy. The topic has received the devoted attention of a large fraction of the LEP experimentalists, with many new techniques and refinements. A good review can be found in Ref. 54. The best quantity to measure is $\mathrm{R}_{b}=\frac{\Gamma_{b}}{\Gamma_{\text {had }}}$, where the $b$-vertex correction is nicely isolated with little theoretical uncertainty. ${ }^{17}$ The methods group in three categories: tagging with leptons, event shape, or displaced vertex.

The oldest technique is $b$-tagging with high $P, P_{\perp}$ leptons. Leptons are identified among all charged tracks in hadronic events and selected on their longitudinal or transverse momentum with respect to the nearest jet. Charm-decay background is separated statistically from a global fit to the lepton distributions. As a result, the $b$ partial width that is extracted this way is strongly correlated with assumptions made on the charm decays. The efficiency is reduced to less than $10 \%$ by (i) the leptonic branching ratio ( $40 \%$ for either $b$ into either electron or muon), and (ii) the high $P, P_{\perp}$ cuts necessary to isolate a pure sample. Typically, a purity of $80 \%$ can be reached with an efficiency of $5 \%$. The heavy flavor analyses using lepton tagging are described in Refs. $55-58$. 
Figure 8: A fully reconstructed example of a $\mathrm{Z} \rightarrow b \bar{b}$ event. Here a $B_{s}$ is identified by its decay $B_{s} \rightarrow \psi^{\prime} \phi$. The $\psi^{\prime}$ decays into two muons and the $\phi$ into two K's. Left, front view $(\mathrm{x}-\mathrm{y})$. Right: expanded view of the vertex showing the VDET hits, and the reconstructed primary and secondary vertices.

Figure 9: Decay length significance distribution in OPAL. The events with forward tags provide the $b$ signal, and those with backward tags a control sample for resolution and light quark background. 
Event-shape analyses have, in principle, the advantage of using all events. Various kinematical variables can be reconstructed in jets that are sensitive to the presence of a heavy, fast object decaying isotropically. An efficient variable is the boosted sphericity product and variations thereof. Such analyses are described in Refs. 59-61.

Vertex tagging is the area where most progress has been accomplished in the last year. The tool of identification is now the long (1.5 ps) lifetime of the $b$ hadrons, associated with their large decay multiplicity. As a consequence, events containing a $b$-quark tend to contain several charged tracks originating from a secondary vertex situated several millimeters from the main interaction point, as can be seen in the beautiful example shown in Fig. 8. To perform this task, the LEP experiments are equipped with high-precision vertex detectors. The LEP experiments have used various characterizations of the detached secondary vertex properties. ALEPH ${ }^{62}$ and DELPHI ${ }^{63}$ have used the product of probabilities of the tracks to extrapolate back to the vertex, while OPAL ${ }^{64}$ selects signal and background control samples on the basis of impact parameter significance $\delta / \sigma(\delta)$, see Fig. 9. The L3 detector is being upgraded to include a vertex detector to be operational in 1994.

Since the production and decay of $b$-hadrons is not very well-known, the tagging efficiency cannot be calculated with certainty. However, there are two b's per $\mathrm{Z} \rightarrow b \bar{b}$ event, and use is made of the double-tag method to measure the tagging efficiency from the data, by comparing the rates of single-tagged and double-tagged events. Only backgrounds and hemisphere correlations have to be calculated from Monte Carlo. The backgrounds come mostly from light quarks which fake the tag. $u, d$, and $s$ quarks can fake a lepton tag because of a misidentified hadron, or the vertex tag because of secondary vertices (strange particle decays or secondary interactions). Charm constitutes a more serious background, as it is a source of prompt leptons and of secondary vertices, albeit with lower multiplicities. The charm background estimates require good knowledge of charm production and are presently the dominant source of systematic error.

The hemisphere correlations are mostly of geometrical nature, since the two $b$ quarks in an event are emitted back-to-back and tend to hit the detector inhomogeneities in a correlated manner. However, there are some physical causes to correlations, such as hard gluon emission that reduces $b$ momenta on both sides 
of an event. Better control of systematics can be obtained by calibrating one of the tagging methods against another (mixed tag). The most precise methods are those using the vertex tag, and the least precise systematically are those using event shape variables. The results are summarized in Table 9. The experimental results in this table have been corrected to a partially harmonized set of input parameters, and the average performed taking into account common sources of errors, by the LEP electroweak working group. ${ }^{48,92}$

\begin{tabular}{|c|lrcc|}
\hline Method & Experiment & \multicolumn{3}{c|}{$\begin{array}{c}\Gamma_{b} \\
\Gamma_{\text {had }}\end{array}$} \\
& & value & exp. error & modeling error \\
\hline High $P, P_{\perp}$ & ALEPH & 0.216 & \pm 0.006 & \pm 0.005 \\
lepton tag & L3 & 0.2187 & \pm 0.008 & \pm 0.008 \\
& OPAL & 0.2252 & \pm 0.011 & \pm 0.007 \\
& DELPHI & 0.2145 & \pm 0.0089 & \pm 0.0066 \\
\hline Event shape & ALEPH (mixed) & 0.228 & \pm 0.0054 & \pm 0.004 \\
variables: & L3 & 0.222 & \pm 0.003 & \pm 0.007 \\
\hline Microvertex tag: & DELPHI & 0.2214 & \pm 0.0020 & \pm 0.0028 \\
& ALEPH & 0.2187 & \pm 0.0022 & \pm 0.0026 \\
& OPAL & 0.2171 & \pm 0.0021 & \pm 0.0021 \\
\hline \hline Average for SM $\frac{\Gamma_{c}}{\Gamma_{\mathrm{had}}}$ & & 0.2192 & & \pm 0.0018 \\
\hline
\end{tabular}

Table 9: $\frac{\Gamma_{b}}{\Gamma_{\mathrm{had}}}$ measurements at LEP. The numbers have been shifted to a common set of parameters and the averages have been computed as described in Refs. 48 and 92. They are, therefore, not necessarily identical to the numbers given by the experiments. Errors that would result from floating the charm partial width are not shown.

The main background to $b$-tag being charm, there is a large correlation between the $b$ and $c$ partial widths. If the measurement of $\frac{\Gamma_{b}}{\Gamma_{\mathrm{had}}}$ is to be interpreted within the SM, $\frac{\Gamma_{c}}{\Gamma_{\mathrm{had}}}$ is essentially fixed to its SM value, even if one lets $\sin ^{2} \theta_{\mathrm{w}}^{\text {eff }}$ and $\delta_{v b}$ float independently of each other. On the other hand, it is also interesting to 
measure $\frac{\Gamma_{b}}{\Gamma_{\mathrm{had}}}$ and $\frac{\Gamma_{c}}{\Gamma_{\mathrm{had}}}$ independently. In this case, the result of Table 9 becomes:

$$
\begin{aligned}
& \frac{\Gamma_{b}}{\Gamma_{\text {had }}}=0.2202 \pm 0.0020, \\
& \frac{\Gamma_{c}}{\Gamma_{\text {had }}}=0.1583 \pm 0.0098,
\end{aligned}
$$

with a correlation of -0.40 between these two numbers.

\section{Measurements of the Effective Weak Mixing Angle}

\section{$5.1 \tau$ Polarization}

In the case of the $\tau$ lepton, the charged decay provides us with a final state polarization analyzer. ${ }^{65,66}$ The ALEPH, ${ }^{67}$ DELPHI, ${ }^{68}$ OPAL, ${ }^{69}$ and L3 (Ref. 21 ) collaborations have presented results for the following five decay channels: $\tau \rightarrow$ $\pi \nu_{\tau}$ (B.R. 12\%), $\tau \rightarrow e \nu_{e} \nu_{\tau}$ (B.R. 18\%), $\tau \rightarrow \mu \nu_{\mu} \nu_{\tau}$ (B.R. 18\%), $\tau \rightarrow \rho \nu_{\tau} \rightarrow$ $\pi^{-} \pi^{0} \nu_{\tau}$ (B.R. 24\%), and $\tau \rightarrow a_{1} \nu_{\tau} \rightarrow \pi^{-} \pi^{+} \pi^{0} \nu_{\tau}$ (B.R. 8\%). The analyses do not distinguish here the nature of the charged hadron; channels with kaons are included as well, but have very similar spin properties.

The extraction of the $\tau$ polarization is illustrated in Fig. 10. For the lepton and $\pi$ channels, all the information is contained in the momentum spectrum of the charged particle, which is fitted to a linear combination of the distributions for positive and negative helicities. For $\rho$ and $a_{1}$ decays, the full information must be retrieved by a full analysis of the decay products, as shown by Rougéf0 and developed in Ref. 71. For the $\tau \rightarrow \rho \nu_{\tau}$ decay, the $\tau$ helicity affects the distributions of both $\tau$ and $\rho$ decay angles in a way that depends on the $\pi^{-} \pi^{0}$ mass. This set of observables, $\{\xi\}$, defines the final state. The probability density functions for the \pm 1 helicity, $W^{ \pm}(\{\xi\})$, are used to build an optimal variable $\omega(\{\xi\})=\left(W^{+}-W^{-}\right) /\left(W^{+}+W^{-}\right)(\{\xi\})$ and fit the $\tau$ polarization. For the $a_{1}$, the decay is defined by six variables. Full use of the density function in this set makes the $a_{1}$ channel more sensitive than the leptonic one.

By analyzing the polarization as a function of polar angle, one can derive both the average $\tau$ polarization, $\mathcal{P}_{\tau}$, and the forward-backward polarization asymmetry $\mathrm{A}_{\mathrm{FB}}^{\mathrm{pol}(\tau)}$, as shown in Fig. 11. The results in the individual channels from the LEP 


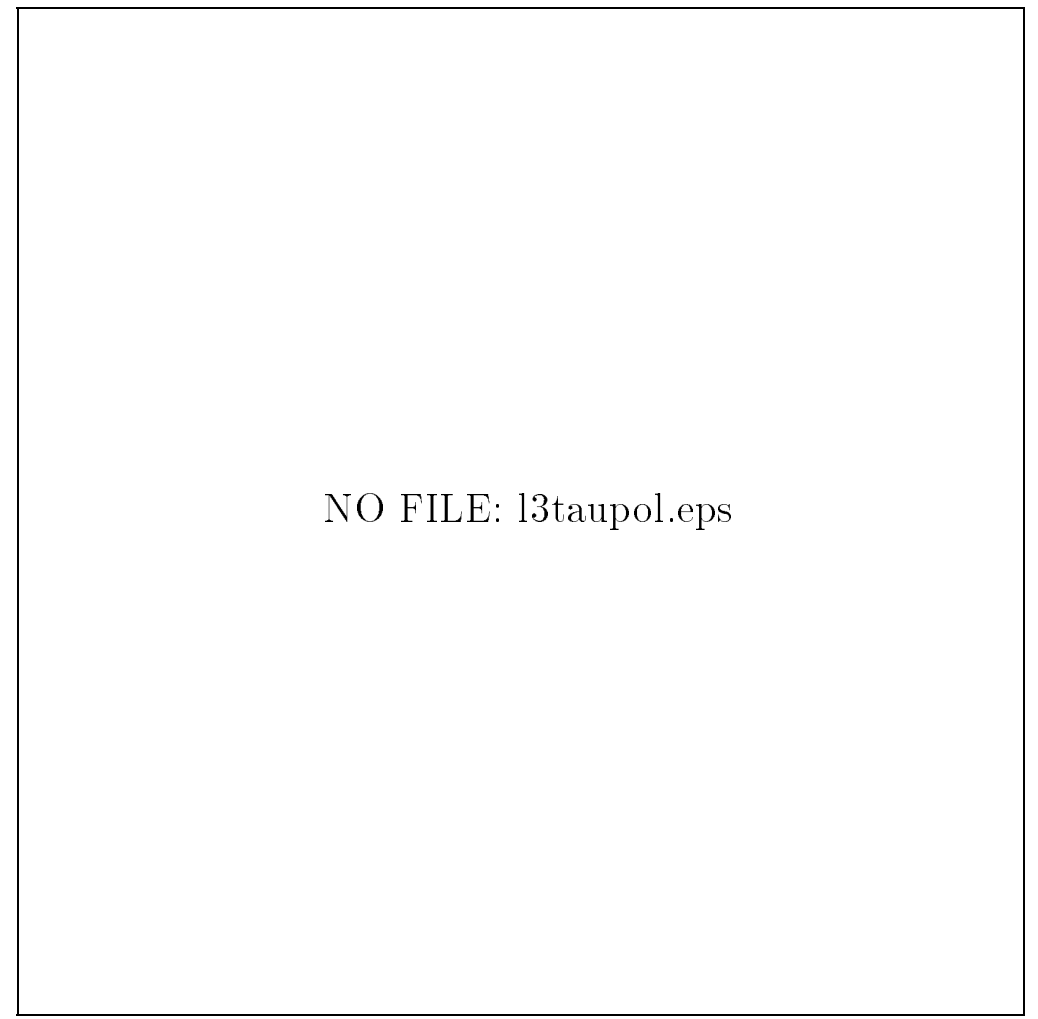

Figure 10: Extraction of the $\tau$ polarization in L3. (a) $\tau \rightarrow \mu \nu_{\mu} \nu_{\tau}$; (b) $\tau \rightarrow e \nu_{e} \nu_{\tau}$; (c) $\tau \rightarrow \pi \nu_{\tau}$; (d) $\tau \rightarrow \rho \nu_{\tau}$. The full line is the result of the fit, which is a linear sum of the components due to positive (dotted line) or negative (dashed line) $\tau$ helicities. 
experiments are summarized in Table 10. The measurements are compatible with each other. It can be seen also that experimental systematic errors on $\mathcal{A}_{\tau}$ are now almost as large as the statistical ones. Improvements will require ingenuity! Systematic errors on $\mathcal{A}_{e}$ are, on the other hand, very small.

\begin{tabular}{|ccccc|}
\hline \hline Channel & ALEPH & DELPHI & OPAL & L3 \\
\hline \hline \multicolumn{7}{c}{$\mathcal{P}_{\tau} \times 10^{3}$} & & \\
$e \nu_{e} \nu_{\tau}$ & $-214 \pm 65 \pm 33$ & $-130 \pm 76 \pm 81$ & $-85 \pm 58 \pm 45$ & $-127 \pm 79 \pm 24$ \\
$\mu \nu_{\mu} \nu_{\tau}$ & $-123 \pm 55 \pm 27$ & $-33 \pm 68 \pm 41$ & $-80 \pm 54 \pm 33$ & $-254 \pm 72 \pm 28$ \\
$\pi \nu_{\tau}$ & $-148 \pm 26 \pm 11$ & $-192 \pm 38 \pm 40$ & $-143 \pm 37 \pm 30$ & $-128 \pm 36 \pm 28$ \\
$\rho \nu_{\tau}$ & $-90 \pm 24 \pm 18$ & $-119 \pm 28 \pm 31$ & $-157 \pm 24 \pm 15$ & $-166 \pm 28 \pm 17$ \\
$a_{1} \nu_{\tau}$ & $-144 \pm 42 \pm 22$ & $-184 \pm 66 \pm 59$ & N.A. & $-250 \pm 128 \pm 34$ \\
\hline \hline \multicolumn{7}{c}{$\mathcal{A}_{\tau} \times 10^{3}$} & & $144 \pm 13 \pm 15$ \\
All & $137 \pm 12 \pm 8$ & $144 \pm 18 \pm 16$ & $153 \pm 19 \pm 13$ & $154 \pm 20 \pm 12$
\end{tabular}

Table 10: Results of the $\tau$ polarization analyses for individual channels from the LEP experiments. For ALEPH and L3 values of individual channels, only 1992 data are shown.

The extraction of $\mathcal{P}_{\tau}$ assumes that the $\tau$ decays through maximally parity violating V-A charged current. The errors in Table 10 do not allow for possible violation of this assumption. It is possible, however, to place constraints on the $\tau$ neutrino helicity $\xi$ by studying the correlation between the helicities of the two $\tau$ 's in an event. The ARGUS ${ }^{72}$ (at DESY) and ALEPH ${ }^{73}$ collaborations have performed such analyses, yielding:

$$
\begin{gathered}
\xi \nu_{\tau}=-1.25 \pm 0.23 \pm_{0.15}^{0.08}(\mathrm{ARGUS}) \text { and } \\
\xi \nu_{\tau}=-0.99 \pm 0.07 \pm 0.04(\mathrm{ALEPH})
\end{gathered}
$$

This confirms beautifully that the $\tau$ family has the same multiplet structure as the electron and muon. If one uses this empirical value for the $\nu_{\tau}$ helicity, a common error of $\Delta \mathcal{P}_{\tau}=0.015$ has to be added to the results of Table 10 . The polarization results can be expressed as a measurement of the $\tau$ and electron 
couplings, or of $\sin ^{2} \theta_{\mathrm{w}}^{\mathrm{eff}}$ :

$$
\begin{aligned}
& \mathcal{A}_{\tau}=0.143 \pm 0.010 \sin ^{2} \theta_{\mathrm{w}}^{\mathrm{eff}}=0.2320 \pm 0.0013 \\
& \mathcal{A}_{e}=0.135 \pm 0.011 \quad \sin ^{2} \theta_{\mathrm{w}}^{\mathrm{eff}}=0.2330 \pm 0.0014
\end{aligned}
$$

The values of $\mathcal{A}_{\tau}$ and $\mathcal{A}_{e}$ are essentially uncorrelated and in good agreement with lepton universality.

\subsection{Light Quark Asymmetries}

In principle, the quark asymmetries $\mathrm{A}_{\mathrm{FB}}^{(q)}$ offer better sensitivity to the measurement of couplings and $\sin ^{2} \theta_{\mathrm{w}}^{\text {eff }}$ than the leptonic forward-backward asymmetries, as well as better event statistics. However, it is difficult to tag specific quark final states and to measure their charge.

Inclusive hadronic charge asymmetry measurements have been carried out by ALEPH, ${ }^{74,75}$ DELPHI, ${ }^{76}$ and OPAL ${ }^{77}$ The method is based on the premise, first suggested by Feynman, ${ }^{78}$ that the original quark charge is carried out by the resulting jet of particles. This property has since been verified in several reactions where the original quark flavor is known, in particular (anti)neutrino or muon deep inelastic scattering. ${ }^{79}$

The method used by ALEPH and DELPHI is described here. OPAL used a different one, based on the three highest momentum particles, with somewhat better statistical sensitivity. Each event is separated in two hemispheres, according to the thrust axis. The momentum-weighted hemisphere charge is constructed:

$$
\mathrm{Q}_{\mathrm{F}, \mathrm{B}}=\frac{\sum_{\mathrm{F}, \mathrm{B}} \mathrm{p}_{\| \mathrm{i}}^{\kappa} \mathrm{q}_{\mathrm{i}}}{\sum_{\mathrm{F}, \mathrm{B}} \mathrm{p}_{\| \mathrm{i}}^{\kappa}}
$$

where $\mathrm{q}_{\mathrm{i}}$ is the charge of particle $\mathrm{i}, \mathrm{p}_{\| \mathrm{i}}$ its momentum projected on the thrust axis, and $\kappa$ is a parameter that is varied for systematic checks. Maximum sensitivity is found for $\kappa=1$. A good estimate of charge for each event is the difference between the forward and backward hemisphere $\mathrm{Q}_{\mathrm{FB}}=\mathrm{Q}_{\mathrm{F}}-\mathrm{Q}_{\mathrm{B}}$.

A significant average charge asymmetry, $\left\langle\mathrm{Q}_{\mathrm{FB}}\right\rangle$, is observed for the inclusive hadronic event sample. The expected charge asymmetry is given by:

$$
\left\langle\mathrm{Q}_{\mathrm{FB}}\right\rangle=\sum_{\text {quark flavors }} \delta_{f} \mathrm{~A}_{\mathrm{FB}}^{(f)} \frac{\Gamma_{f}}{\Gamma_{\text {had }}},
$$


where $\delta_{f}$, the charge separation, is the average charge difference between the quark and antiquark hemisphere: $\delta_{f}=\left\langle\mathrm{Q}_{f}-\mathrm{Q}_{\bar{f}}\right\rangle$. The forward-backward asymmetries, see Eq. (5), are all positive, but the signs of the charge separations are different. This results in a large cancellation, already at parton level.

The experimental uncertainties are small, but the interpretation of $\left\langle Q_{\mathrm{FB}}\right\rangle$ in terms of $\sin ^{2} \theta_{\mathrm{w}}^{\mathrm{eff}}$ is affected by the uncertainty in the calculation of the charge separations. This is estimated by varying the parameters of the hadronization models, and by comparing various models. The experiments find different values for the charge separations, which can be traced to a different choice of input parameters in the simulation, and of the decay tables for heavy flavored particles. The most critical parameters in the simulation of light quark charges are those controlling pair production of strange particles and baryon pairs. Improved understanding of particle composition and correlations in jets will help in reducing these errors. The decay tables of heavy particles will remain incompletely known, and the solution is probably to measure directly the heavy quark jet charges by means of tagged events.

ALEPH has presented a preliminary analysis, ${ }^{75}$ where the fragmentation systematic error is reduced by using a direct measurement of the $b$-jet charge, based on lepton and lifetime tagged $b$ samples, as well as a constraint obtained by measuring the quantity $\left\langle\mathrm{Q}_{\mathrm{F}} \cdot \mathrm{Q}_{\mathrm{B}}\right\rangle$. It can easily be shown that, for a sample consisting only of one type of quark $f$, and up to small correlation terms,

$$
\left\langle\mathrm{Q}_{\mathrm{F}} \cdot \mathrm{Q}_{\mathrm{B}}\right\rangle \simeq-\frac{\delta_{f}^{2}}{4}
$$

This method can be used for a selected $b$ sample to measure $\delta_{b}$. In the inclusive sample, $\left\langle Q_{F} \cdot Q_{B}\right\rangle$ measures a weighted sum of the squares of the charge separations but still constrains usefully some of the fragmentation parameters (unfortunately, not strange particles and baryon pair production!). The results are expressed in terms of $\sin ^{2} \theta_{\mathrm{w}}^{\text {eff }}$. QCD corrections and $B^{0}$ mixing are automatically taken into account by the method. The present status is given in Table 11 .

By selecting events with a fast $K^{ \pm}$and $\Lambda$, an enriched sample of signed $s \bar{s}$ events can be obtained. This is possible in DELPHI thanks to the particle identification provided by the Ring Imaging Cherenkov. ${ }^{80}$ The mass of a particle of momentum $P$ is measured by the Cherenkov angle $\theta_{c}$ as: $m=P . n \cdot \cos \theta_{c}$. Kaons are well-separated for momentum between 10 and $18 \mathrm{GeV}$, as shown in Fig. 12. 


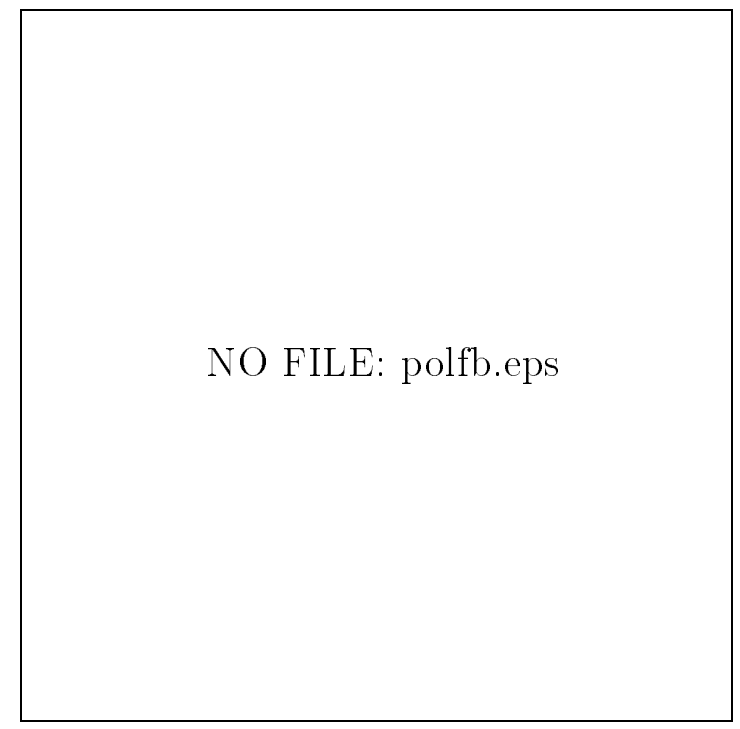

Figure 11: Angular dependence of the $\tau$ polarization in ALEPH. The lines show the result of a fit to Eq. (6) in case one does (dotted line) or does not (full line) assume lepton universality.

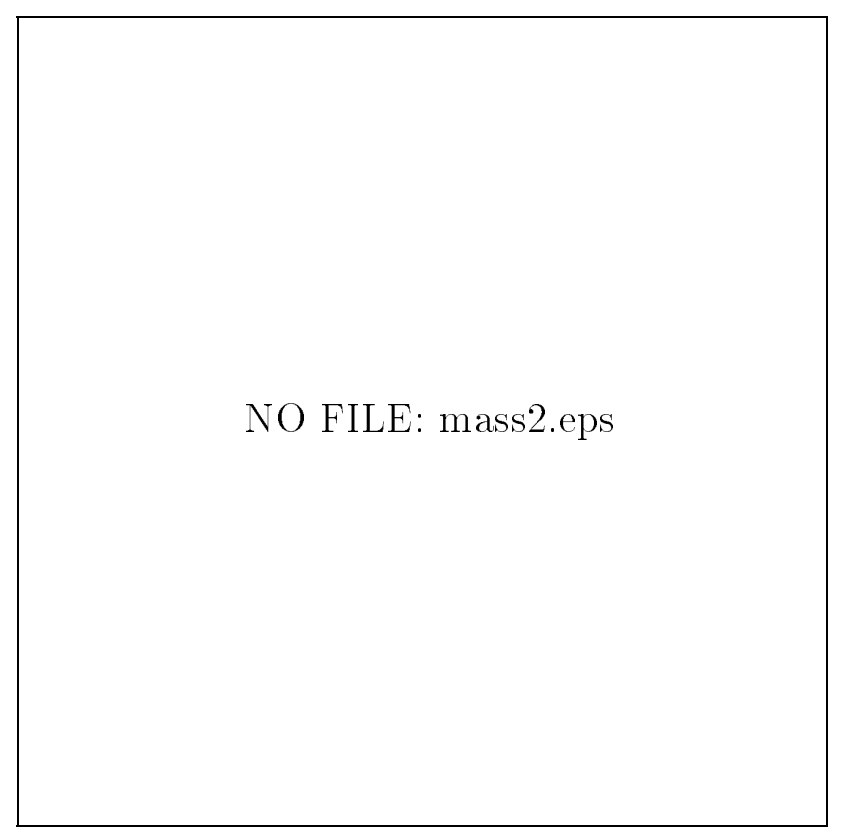

Figure 12: Mass reconstruction with the DELPHI RICH. 


\begin{tabular}{|l|c|c|c|c|c|c|}
\hline \hline Expt. & Status & $s / u$ & EW Prog. & QCD & Cuts & $\sin ^{2} \theta_{\mathrm{w}}^{\text {eff }}$ \\
\hline \hline ALEPH & $89-90$ publ. & & & & & \\
& $89-92$ prel. & $0.315 \pm 0.045$ & EXPOSTAR & No & No & $0.2317 \pm 0.0013 \pm 0.0011$ \\
\hline DELPHI & $90-91$ publ. & $0.315 \pm 0.045$ & ZFITTER & Yes & No & $0.2345 \pm 0.0030 \pm 0.0027$ \\
\hline OPAL & $90-91$ publ. & $0.285 \pm 0.050$ & ZFITTER & Yes & S $<0.12$ & $0.2321 \pm 0.0017 \pm 0.0028$ \\
\hline \hline Average & & & & & & $0.2320 \pm 0.0011 \pm 0.0011$
\end{tabular}

Table 11: Summary of the determination of $\sin ^{2} \theta_{\mathrm{w}}^{\mathrm{eff}}$ from inclusive hadronic charge asymmetries at LEP.

The expected purity for this mass range is $42 \%$ of $s$ quarks of the right sign, and the expected asymmetry is -0.04. A significant asymmetry is measured, see Fig. 13. Similar, though less precise, analyses are obtained with $\Lambda$ 's. Fast $K^{0}$ and neutrons, detected in the hadron calorimeter, provide an unsigned tag for $s \bar{s}$ and $d \bar{d}$ events. This can be combined with jet charge to measure the downquark asymmetry. The measured asymmetries ${ }^{81}$ are consistent with the SM value 0.0937 :

$$
\begin{array}{rll}
K^{ \pm}: & 0.118 & \pm 0.031_{\text {stat. }} \pm 0.016_{\text {syst }} \\
\Lambda: & 0.135 & \pm 0.055_{\text {stat. }} \pm 0.037_{\text {syst. }} \\
K^{0}, n: & 0.111 & \pm 0.031_{\text {stat. }}^{+0.068}{ }_{-0.054}^{+0.5 s t .}
\end{array}
$$

\subsection{Heavy Quark Asymmetries}

The asymmetry can also be measured for individual quark species if one is able to:

- Tag the specific quark flavor. This can be done for $b$ and $c$ quarks by means of their semileptonic decays, which produce prompt leptons. High $P_{\perp}$ leptons $\operatorname{tag} b$ quarks; low $P_{\perp}$ leptons are enriched in $c$ quarks. Another possibility for $b$ 's is the lifetime tag and for $c$ the recognition of a high-momentum $D$ meson.

- Measure the scattering angle. The thrust axis of the event is usually used as a measure of the original quark-antiquark direction before fragmentation. 
- Assign an orientation to the event axis defined above. In the selection with leptons or with $D$ mesons, the sign of the lepton or the flavor of the meson can be used. In case the event is recognized by lifetime tag, one can use the jet charge (as in the inclusive sample) to measure in a statistical way the asymmetry.

All LEP collaborations have performed an analysis using lepton tagging. .5, $82-84^{-8}$ There, the prompt lepton samples are analyzed in a global way to extract simultaneously the $b$ and $c$ partial width, the direct and cascade semileptonic branching fractions, the inclusive $B^{0}-\overline{B^{0}}$ mixing, and the $b$ and $c$ asymmetries. The asymmetry has to be corrected for QCD radiation ${ }^{85}$ (3\% correction), and for the experimental effects associated with the event axis determination and its orientation with a lepton (a few\%).

ALEPH, ${ }^{86}$ DELPHI, ${ }^{87}$ and OPAL ${ }^{88}$ have presented the asymmetry measured from jet-charge in the lifetime-tagged lepton sample. The weakness of the inclusive jet charge asymmetry described above, namely the dependence upon fragmentation parameters, is avoided by determining the $b$ charge from the data using the charge correlation between opposite hemispheres, Eq. (18). As mentioned before, no QCD correction is necessary with this method.

The charm asymmetry is also measured with $D$ mesons by ALEPH, ${ }^{89}$ DELPHI, ${ }^{90}$ and OPAL. ${ }^{91}$

The averaging of these results is a very delicate enterprise. A preliminary procedure is attempted by the LEP electroweak working group. ${ }^{48,92}$ The results are corrected for photonic effects to obtain the pole asymmetries:

$$
\begin{aligned}
& \mathrm{A}_{\mathrm{FB}}^{(b)}{ }^{0}=0.0967 \pm 0.0038 \\
& \mathrm{~A}_{\mathrm{FB}}^{(c)}=0.0760 \pm 0.0091 .
\end{aligned}
$$

The correlation between these numbers is 0.08 . They can be expressed as measurements of $\sin ^{2} \theta_{\mathrm{w}}^{\text {eff }}$ :

$$
\begin{aligned}
& \sin ^{2} \theta_{\mathrm{w}}^{\mathrm{eff}}=0.23268 \pm 0.00068(\text { from b) } \\
& \left.\sin ^{2} \theta_{\mathrm{w}}^{\mathrm{eff}}=0.2310 \pm 0.0021 \text { (from } \mathrm{c}\right) .
\end{aligned}
$$

The $b$ forward-backward polarized asymmetry defined in Eq. (8) can be measured if the beams are polarized. This has recently been done at SLC, ${ }^{93}$ both 
with lepton tag and the combined vertex-tag/jet charge method. For $5 \times 10^{4}$ $\mathrm{Z}$ decays with $63 \%$ polarization, one finds:

$$
\mathrm{A}_{\mathrm{FB}}^{\mathrm{pol}(b)}=\mathcal{A}_{b}=0.93 \pm 0.14
$$

From LEP data, combining the measurement of $\mathrm{A}_{\mathrm{FB}}^{(b)}{ }^{0}=\frac{3}{4} \mathcal{A}_{e} \mathcal{A}_{b}$ and the value $\mathcal{A}_{e}=0.135 \pm 0.011$ obtained from leptonic asymmetries and $\tau$ polarization (see below), one can derive $\mathcal{A}_{b}=0.955 \pm 0.086$. The two values are consistent with the SM expectation $\mathcal{A}_{b}=0.94$.

\subsection{Summary on $\sin ^{2} \theta_{\mathrm{w}}^{\text {eff }}$}

The different values of $\sin ^{2} \theta_{\mathrm{w}}^{\text {eff }}$ measured from asymmetries and $\tau$ polarization at LEP and $\mathrm{A}_{\mathrm{LR}}$ at $\mathrm{SLC}^{94}$ are summarized in Fig. 14. Although one can extract a value of $\sin ^{2} \theta_{\mathrm{w}}^{\text {eff }}$ from other measurements in the framework of the minimal $\mathrm{SU}(2)_{\mathrm{L}} \times \mathrm{U}(1)$ model, in particular, from $\Gamma_{e}$ or $\mathrm{M}_{\mathrm{W}}$, asymmetries provide the quantity that corresponds exactly to the definition of Eq. (9). The measurements presented above average to:

$$
\sin ^{2} \theta_{\mathrm{w}}^{\mathrm{eff}}=0.23167 \pm 0.00040 .
$$

This number will play an important role in the determination of electroweak radiative effects.

\section{Analysis of Electroweak Measurements}

\subsection{Lepton Universality}

The couplings of the leptons can be extracted from the measurements of $\Gamma_{e}, \Gamma_{\mu}$, $\Gamma_{\tau}, \mathrm{A}_{\mathrm{FB}}^{(e)}, \mathrm{A}_{\mathrm{FB}}^{(\mu)}, \mathrm{A}_{\mathrm{FB}}^{(\tau)}, \mathrm{A}_{\mathrm{FB}}^{\mathrm{pol}(\tau)}$, and $\mathcal{P}_{\tau}$. The results of the fit are shown in Table 12. Lepton universality is well-verified and will be assumed in the following.

\subsection{Neutrino Partial Width}

What the experiment really measures is the ratio of the invisible width to the leptonic width:

$$
\frac{\Gamma_{\text {inv }}}{\Gamma_{\ell}}=\left(\sqrt{\frac{12 \pi \mathrm{R}_{\ell}}{\mathrm{M}_{Z}^{2} \sigma_{\text {had }}^{\text {peak }}, 0}}-\mathrm{R}_{\ell}-3\right) .
$$




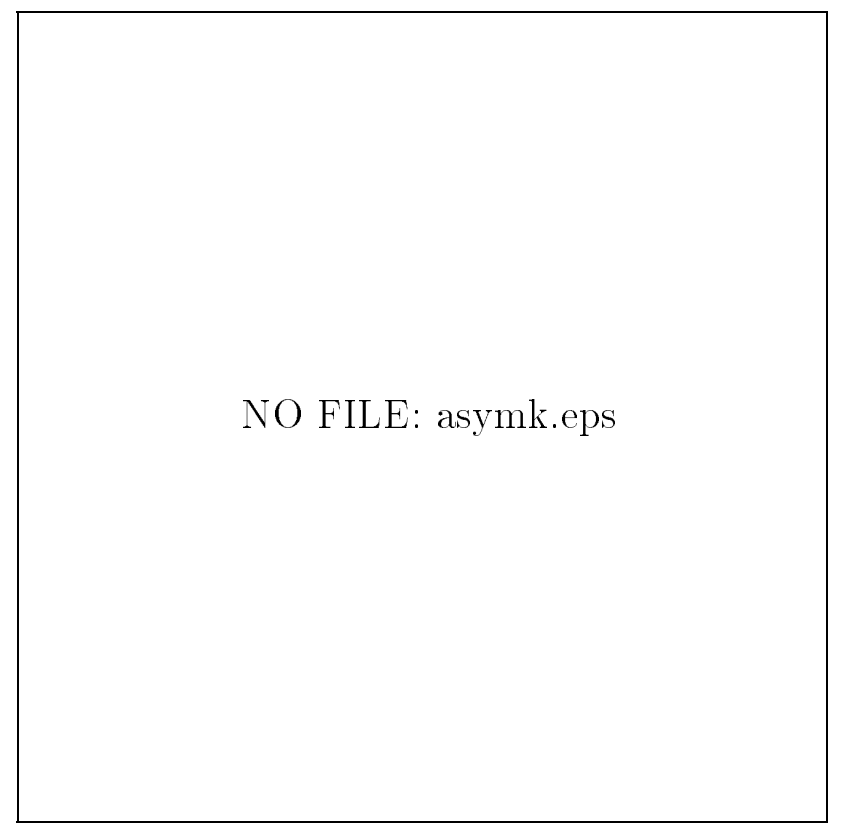

Figure 13: Measured charged $K$ asymmetry in DELPHI.

Figure 14: Summary of measurements of $\sin ^{2} \theta_{\mathrm{w}}^{\mathrm{eff}}$ from the forward-backward asymmetries of leptons, $\tau$ polarization, inclusive quarks, heavy quark asymmetry, and the SLC polarization asymmetry. Also shown is the SM prediction as a function of $\mathrm{M}_{\mathrm{t}}$. 


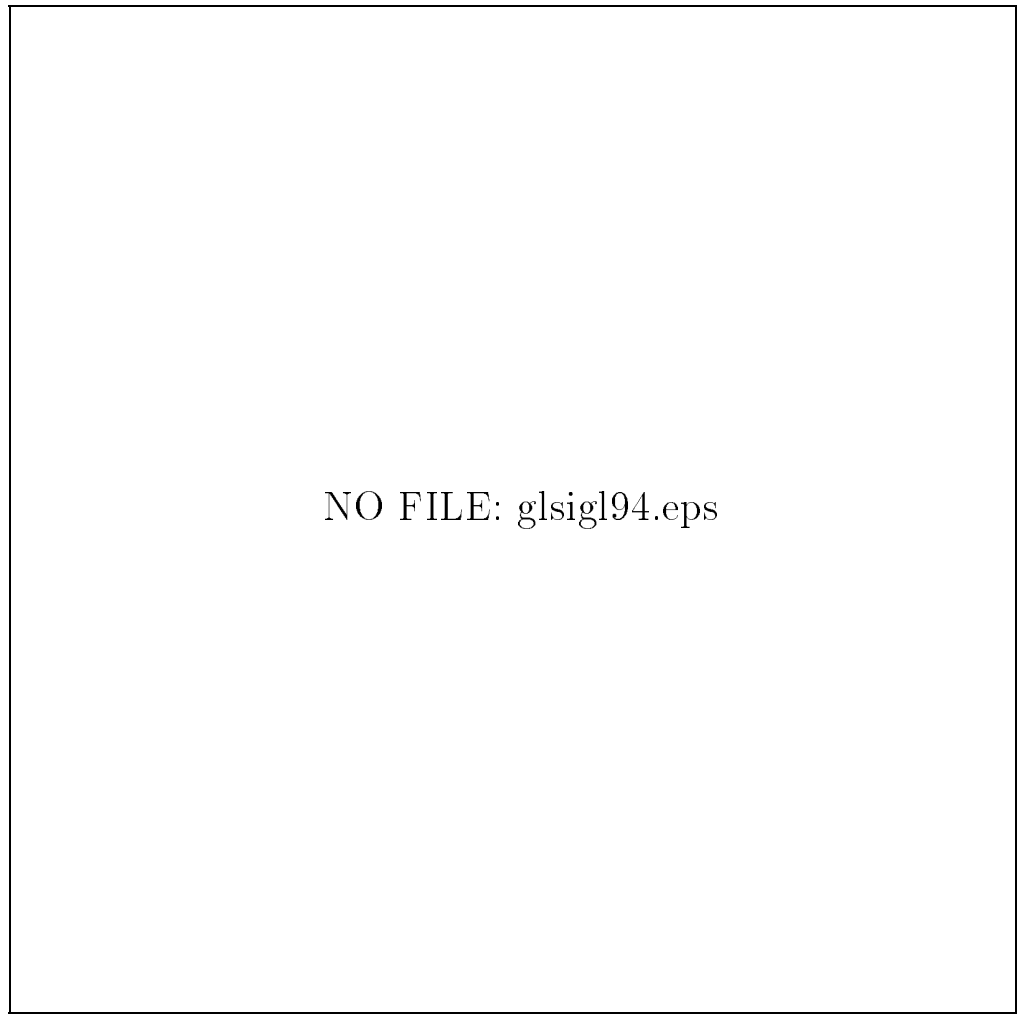

Figure 15: Contours of constant $\chi^{2}$ for $\sin ^{2} \theta_{\mathrm{w}}^{\mathrm{eff}}$ versus $\Gamma_{\ell}$. The $S M$ predictions as a function of $\mathrm{M}_{\mathrm{t}}$ and $\mathrm{M}_{\mathrm{H}}$ are shown. 


\begin{tabular}{|c|c|c|}
\hline & $g_{V \ell}$ & $g_{A \ell}$ \\
\hline$e$ & $-0.0370 \pm 0.0021$ & $-0.50093 \pm 0.00064$ \\
$\mu$ & $-0.0308 \pm 0.0051$ & $-0.50164 \pm 0.00096$ \\
$\tau$ & $-0.0386 \pm 0.0023$ & $-0.5026 \pm 0.0010$ \\
\hline Lepton & $-0.0366 \pm 0.0013$ & $-0.50128 \pm 0.00054$ \\
\hline
\end{tabular}

Table 12: Lepton couplings $g_{V \ell}$ and $g_{A \ell}$ extracted from leptonic asymmetries and $\tau$ polarization, showing the validity of lepton universality.

In $\mathrm{SU}(2)_{\mathrm{L}} \times \mathrm{U}(1)$, the ratio $\Gamma_{\ell} / \Gamma_{\nu}$ can be written as:

$$
\frac{\Gamma_{\ell}}{\Gamma_{\nu}}=\frac{1}{2}\left(1+\left(\frac{g_{V \ell}}{g_{A \ell}}\right)^{2}\right)\left(1+\frac{3 \alpha}{4 \pi}\right)\left(1+\delta_{v}\right)
$$

where $\delta_{v}=-0.0027 \pm 0.0003$ is a vertex correction with no dependence on heavy physics. The ratio $\left(g_{V \ell} / g_{A \ell}\right)^{2}=0.0053 \pm 0.0001$ can be obtained from the measured asymmetries. This yields the prediction:

$$
{\frac{\Gamma_{\ell}}{\Gamma_{\nu}}}^{\text {predicted }}=0.5022 \pm 0.0001
$$

The number of neutrinos is then:

$$
\mathrm{N}_{\nu}={\frac{\Gamma_{\mathrm{inv}}}{\Gamma_{\ell}}}^{\text {measured }} \times{\frac{\Gamma_{\ell}}{\Gamma_{\nu}}}^{\text {predicted }}=2.988 \pm 0.023 .
$$

One can also assume that the number of neutrinos is three and determine the neutrino partial width, a sensitive test of $\mathrm{SU}(2)_{\mathrm{L}} \times \mathrm{U}(1)$ :

$$
\frac{\Gamma_{\ell}}{\Gamma_{\nu}}=0.4992 \pm 0.0038
$$

in excellent agreement with the prediction of Eq. (24). In the following, the number of neutrinos will be fixed to three and the $\mathrm{SU}(2)_{\mathrm{L}} \times \mathrm{U}(1)$ structure assumed.

\subsection{Determination of $\mathrm{SU}(2)_{\mathrm{L}} \times \mathrm{U}(1)$ Radiative Effects}

The LEP measurements contain enough information to extract $\Delta \rho, \delta_{v b}$, and $\Delta_{3 Q}$ defined in Eq. (10). The resulting values for $\Delta \rho, \delta_{v b}$, and $\Delta_{3 Q}$ are shown in Table 13, and compared with the SM predictions. For predicting the hadronic partial width, the value of the QCD coupling constant was constrained to $\alpha_{s}=$ $0.123 \pm 0.006$ in the following. One notes: (i) the sensitivity of the determination 
of $\Delta_{3 Q}$ on the input value of $\Delta \alpha$, and as can be seen from the first one of Eq. (10); and (ii) the sensitivity of the determination of $\delta_{v b}$ on $\alpha_{s}$; as pointed out in Ref. 17 , this comes from the fact the $\mathrm{R}_{\ell}$ is as powerful in determining $\delta_{v b}$ as is $\frac{\Gamma_{b}}{\Gamma_{\text {had }}}$. This point is illustrated in Figs. 16 and 17.

In the SM, $\delta_{v b}$ depends on $\mathrm{M}_{\mathrm{t}}$ only (quadratically), and $\Delta \rho$ depends on $\mathrm{M}_{\mathrm{t}}$ (quadratically) and on $\mathrm{M}_{\mathrm{H}}$ (logarithmically), while $\Delta_{3 Q}$ has a logarithmic dependence on both $M_{t}$ and $M_{H}$, and thus is relatively more sensitive to $M_{H}$. The leading terms are:

$$
\begin{aligned}
\Delta \rho & \simeq \frac{\alpha}{\pi} \frac{\mathrm{M}_{\mathrm{t}}^{2}}{\mathrm{M}_{\mathrm{Z}}^{2}}-\frac{\alpha}{4 \pi} \ln \frac{\mathrm{M}_{\mathrm{H}}^{2}}{\mathrm{M}_{\mathrm{Z}}^{2}}, \\
\Delta_{3 Q} & \simeq \frac{\alpha}{9 \pi} \ln \frac{\mathrm{M}_{\mathrm{H}}^{2}}{\mathrm{M}_{\mathrm{Z}}^{2}} \\
\delta_{v b} & \simeq-\frac{20}{13} \frac{\alpha}{\pi}\left(\frac{\mathrm{M}_{\mathrm{t}}^{2}}{\mathrm{M}_{\mathrm{Z}}^{2}}+\frac{13}{6} \ln \frac{\mathrm{M}_{\mathrm{t}}^{2}}{\mathrm{M}_{\mathrm{Z}}^{2}}\right) .
\end{aligned}
$$

\begin{tabular}{|c|c|c|c|c|c|c|}
\hline Parameter & $\begin{array}{c}\text { Fit } \\
\text { Value }\end{array}$ & $\begin{array}{l}\text { Err } \\
\Delta \alpha\end{array}$ & $\begin{array}{l}\text { Due to } \\
\Delta \alpha_{s}\end{array}$ & \begin{tabular}{||c} 
SM \\
value
\end{tabular} & $\begin{array}{l}\text { Vari } \\
\mathrm{M}_{\mathrm{t}}\end{array}$ & $\begin{array}{c}\text { ation } \\
\mathrm{M}_{\mathrm{H}}\end{array}$ \\
\hline$\Delta \rho \times 10^{4}$ & $33 \pm 19$ & - & $\mp 4$ & 44 & $\begin{array}{l}+15 \\
-14\end{array}$ & $\begin{array}{l}-13 \\
+8\end{array}$ \\
\hline$\Delta_{3 Q} \times 10^{4}$ & $-39 \pm 23$ & \pm 9 & \pm 3 & -60 & ${ }_{-2}^{+1}$ & $\begin{array}{l}-8 \\
+17\end{array}$ \\
\hline$\delta_{v b} \times 10^{4}$ & $-97 \pm 67$ & - & $\mp 51$ & -175 & $\begin{array}{l}-29 \\
+27\end{array}$ & $\begin{array}{l}-1 \\
+1\end{array}$ \\
\hline
\end{tabular}

More complete expressions have been calculated and implemented in computer codes, see Ref. 95.

Table 13: Radiative corrections determined from LEP data. The SM values are given for $\mathrm{M}_{\mathrm{t}}=174 \pm 16 \mathrm{GeV}$ and $\mathrm{M}_{\mathrm{H}}=300_{-240}^{+700} \mathrm{GeV}$. The fit assumes $\alpha_{s}=0.123 \pm 0.006$.

Assuming the SM variation of $\delta_{v b}$ upon $\mathrm{M}_{\mathrm{t}},{ }^{53}$ it can be used $^{10}$ to place a limit on the top quark mass $\mathrm{M}_{\mathrm{t}}<195 \mathrm{GeV}$ at $95 \%$ C.L. This limit is as good at present as what can be obtained in the minimal SM, from $\Delta \rho$ mostly, but less sensitive to cancellation with other new physics. 
Figure 16: Comparison of $\sin ^{2} \theta_{\mathrm{w}}^{\text {eff }}$ and $\frac{\Gamma_{b}}{\Gamma_{\mathrm{had}}}$ and the $S M$ prediction. The constraint from $\mathrm{R}_{\ell}$, assuming $\alpha_{s}=0.123 \pm 0.006$, is an oblique band. 
Figure

17:

Same as Fig. 16, result of a two-parameter fit of $\left\{\frac{\Gamma_{b}}{\Gamma_{\mathrm{had}}}, \mathrm{R}_{\ell}, \sigma_{\mathrm{had}}^{\text {peak }, 0}, \sin ^{2} \theta_{\mathrm{w}}^{\mathrm{eff}}, \Gamma_{\mathrm{Z}}\right\}$ to $\left\{\delta_{v b}, \sin ^{2} \theta_{\mathrm{w}}^{\mathrm{eff}}\right\}$ assumed independent. 
Given the small range allowed for $\Delta_{3 Q}$ in the SM, its measurement constitutes a $0.3 \%$ test at the one-loop level. This test comes mostly from the comparison between the measurements of $\Gamma_{\ell}$ and of $\sin ^{2} \theta_{\mathrm{w}}^{\text {eff }}$ from the asymmetries, shown in Fig. 15. In nonminimal theories, $\Delta_{3 Q}$ provides a test of the Higgs sector. Such a scenario is discussed in Ref. 8, where the related quantity $S \simeq-\Delta_{3 Q} \times 4 \sin ^{2} \theta_{\mathrm{w}}^{\text {eff }} \cos ^{2} \theta_{\mathrm{w}}^{\text {eff }} / \alpha$ is expected to be increased by 2.1 for one generation of technifermions in $N_{c}=4$ technicolor. The corresponding change of $\Delta_{3 Q}$ is -0.024 and is clearly ruled out by LEP experiments.

In principle, the measurement of $\Delta_{3 Q}$ can be used to set limits on the Higgs boson mass and/or on whatever plays its role, in particular on supersymmetrysuch an analysis has been performed, e.g., Refs. 9 and 96 . It can be seen from Fig. 15 that the experimental error is still of the same size as the variation of $\Delta_{3 Q}$ from the Higgs boson mass.

The agreement of the determination of the radiative corrections with the SM predictions is striking, especially if one takes into account the first possible direct determination of $\mathrm{M}_{\mathrm{t}}$ by $\mathrm{CDF},{ }^{97} \mathrm{M}_{\mathrm{t}}=174 \pm 16 \mathrm{GeV}$. This is shown in Figs. 15, 16, and 17 where the three experimentally independent observables $\sin ^{2} \theta_{\mathrm{w}}^{\mathrm{eff}}, \frac{\Gamma_{b}}{\Gamma_{\mathrm{had}}}, \Gamma_{\ell}$ are plotted against each other.

The discovery of the top quark, if confirmed, is an event of considerable importance. First, it happened in the range of masses predicted by the SM from precision measurements. Second, the SM loses one unmeasured free parameter, the only one left being the Higgs mass!

\subsection{Determination of the Top Quark and Higgs Boson Masses}

Having shown the consistency of the measurements with the SM, it is justified to consider all available measurements as measures of $\mathrm{M}_{\mathrm{t}}$, and possibly, $\mathrm{M}_{\mathrm{H}}$.

The most precise measurements to date are: (i) the measurement of the neutrino $\mathrm{NC} / \mathrm{CC}$ ratio from the $\mathrm{CDHS},{ }^{98} \mathrm{CHARM}^{99}$ and $\mathrm{CCFR}^{100}$ experiments, averaging to $\sin ^{2} \theta_{\mathrm{w}}=0.2253 \pm 0.0047$; (ii) the measurement of the $\mathrm{W}$ mass, $\mathrm{M}_{\mathrm{W}}=80.23 \pm 0.18$ (Ref. 101) from UA2, ${ }^{102} \mathrm{CDF},{ }^{103}$ and $\mathrm{D} \emptyset$ (Ref. 104); and (iii) the LEP measurements of the line-shape parameters, $\frac{\Gamma_{b}}{\Gamma_{\text {had }}}$, and of $\sin ^{2} \theta_{\mathrm{w}}^{\text {eff }}$ 
from asymmetries. A fit to these 14 observables yields

$$
\begin{array}{lcl}
\mathrm{M}_{\mathrm{t}}=159 \pm 12 & \left(\mathrm{M}_{\mathrm{H}}=60\right) & \chi^{2}=13.3 \\
\mathrm{M}_{\mathrm{t}}=178 \pm 11 & \left(\mathrm{M}_{\mathrm{H}}=300\right) & \chi^{2}=15.1 \\
\mathrm{M}_{\mathrm{t}}=196 \pm 10 & \left(\mathrm{M}_{\mathrm{H}}=1000\right) & \chi^{2}=16.9
\end{array}
$$

Although the light Higgs mass seems to be preferred, the difference between the

light and heavy Higgs hypothesis is only $\Delta \chi^{2}=3.6$, and no significant limit can be placed. The constraints on $\mathrm{M}_{\mathrm{t}}, \mathrm{M}_{\mathrm{H}}$ placed by this fit are shown in Fig. 18. If the experimental lower limits on $\mathrm{M}_{\mathrm{H}}>63 \mathrm{GeV}$ and $\mathrm{M}_{\mathrm{t}}>131 \mathrm{GeV}$ are included, the situation is not substantially modified, see Fig. 19. Even when the possible direct measurement is included, the upper limit on the Higgs mass improves slightly but not dramatically, Fig. 20. The $\chi^{2}$ difference between $M_{H}=60$ and $\mathrm{M}_{\mathrm{H}}=1000 \mathrm{GeV}$ becomes 4.2 . Of course, this occurs because (i) the values of $\mathrm{M}_{\mathrm{t}}$ from precision measurement and the direct one are so close; and (ii) the error on the top mass from CDF is as large as the variation upon the Higgs mass of the indirect top mass determination from precision measurements.

\section{Conclusions and Outlook}

The advent of the LEP accelerator and experiments has allowed new and precise tests of the SM to be performed. Electroweak measurements have shown no deviation from the minimal picture at the $10^{-3}$ level. This is confirmed by the lack of observation of new particles within the range kinematically reachable, and of the Higgs boson up to $63 \mathrm{GeV}$. The top quark mass was predicted right where it has possibly been found.

So, is there a future for LEP precision experiments?

The first, obvious missing piece is a precision measurement of $M_{t}$. It seems possible that its mass will be measured at the Tevatron with a precision of $\pm 5 \mathrm{GeV}$. Secondly, improvements in electroweak measurements are still to come: (i) The measurement of the $\mathrm{W}$ mass from the energy upgrade of LEP, with a precision of about $30 \mathrm{MeV}$ or better. (ii) It is expected that the statistics of LEP experiments will have increased by a factor of two to five by the end of 1995. The measurement of the $\mathrm{Z}$ mass and width should improve significantly by steady scanning of the $\mathrm{Z}$ resonance, together with precise energy calibration of 


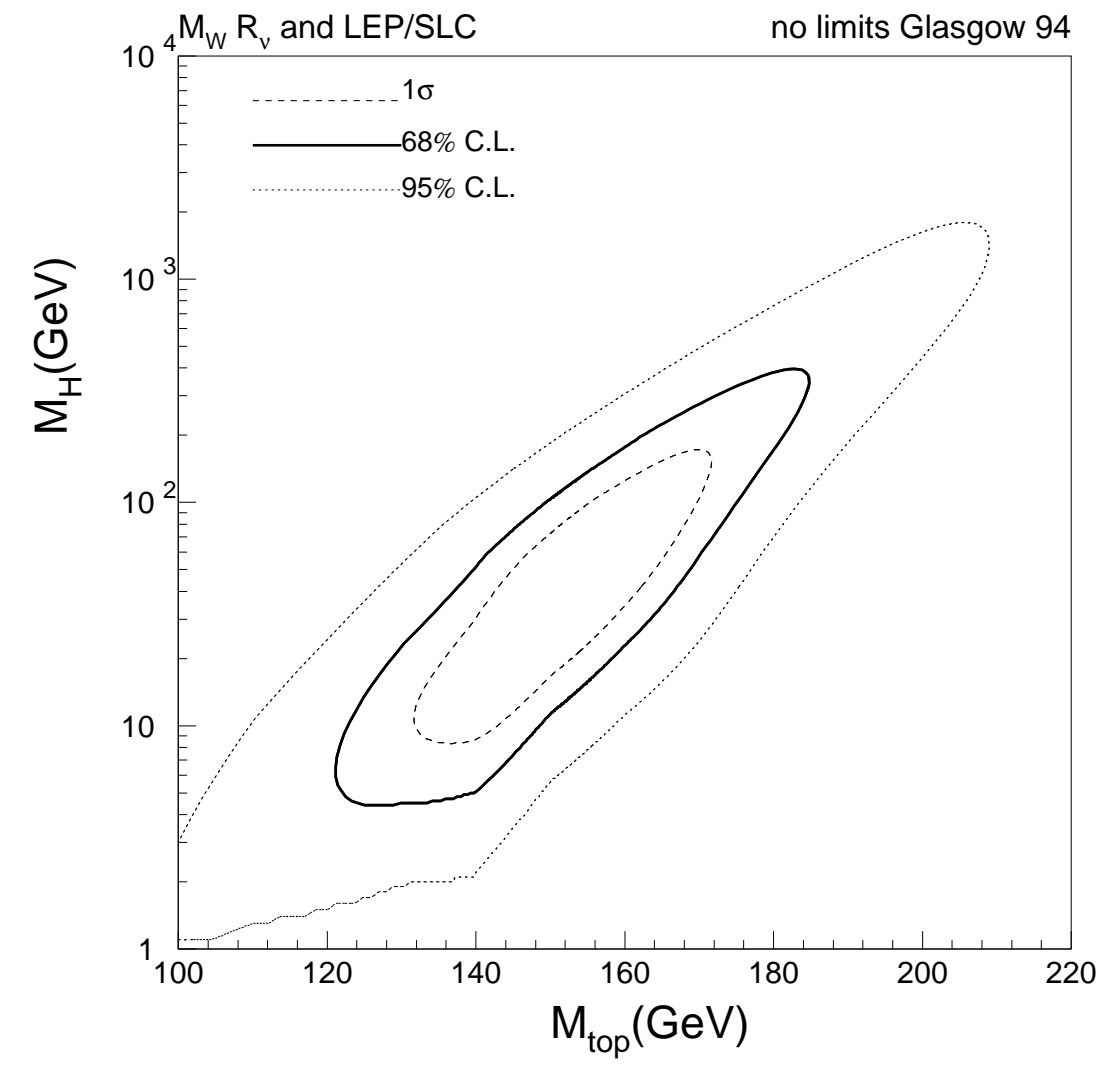

Figure 18: Contours of constant $\chi^{2}$ for the global fit to $\mathrm{M}_{\mathrm{t}}, \mathrm{M}_{\mathrm{H}}$; electroweak measurements only. 


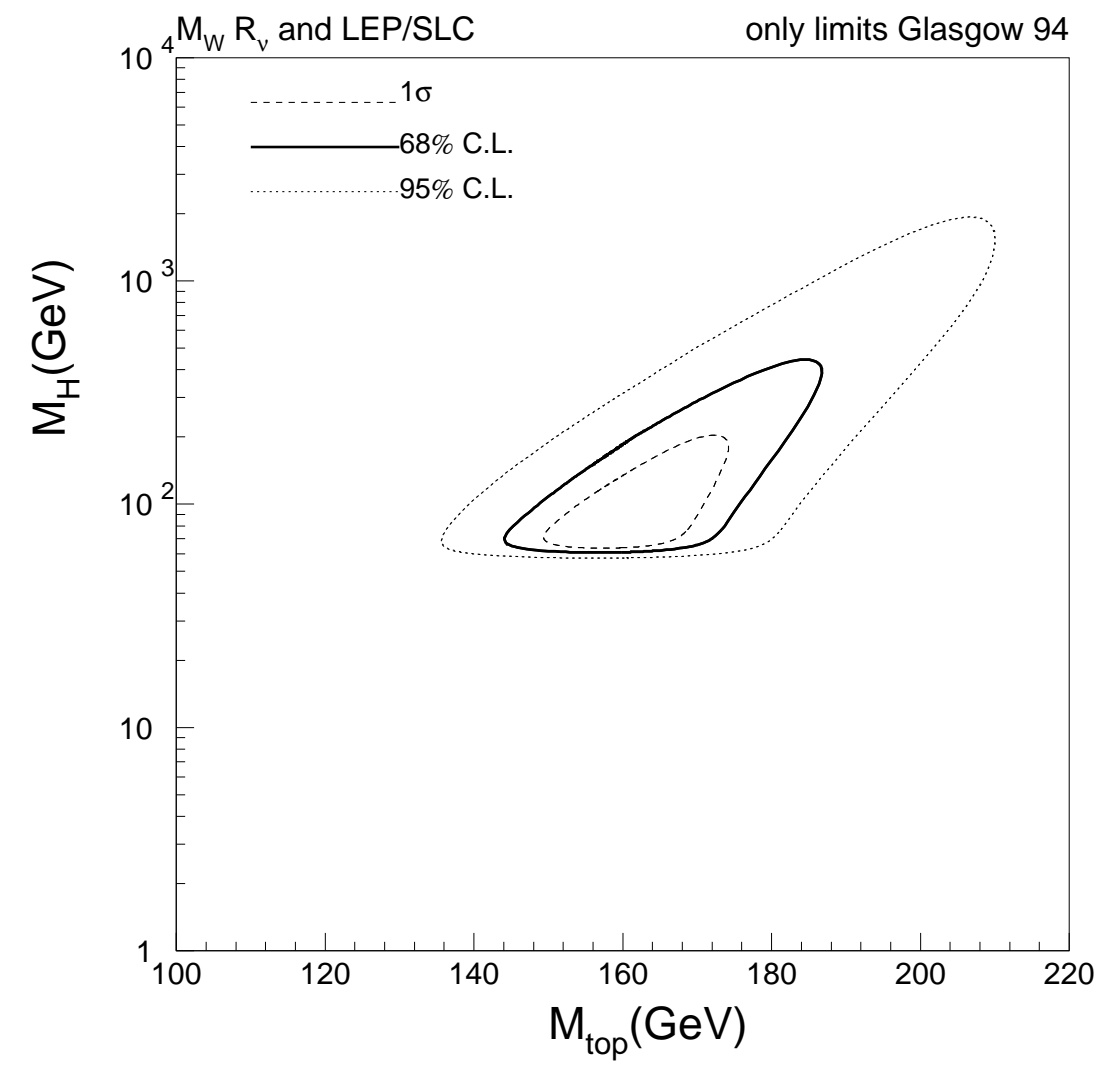

Figure 19: Same as Fig. 18 with direct limits $\mathrm{M}_{\mathrm{t}}>131 \mathrm{GeV}, \mathrm{M}_{\mathrm{H}}>63.5 \mathrm{GeV}$ included. 


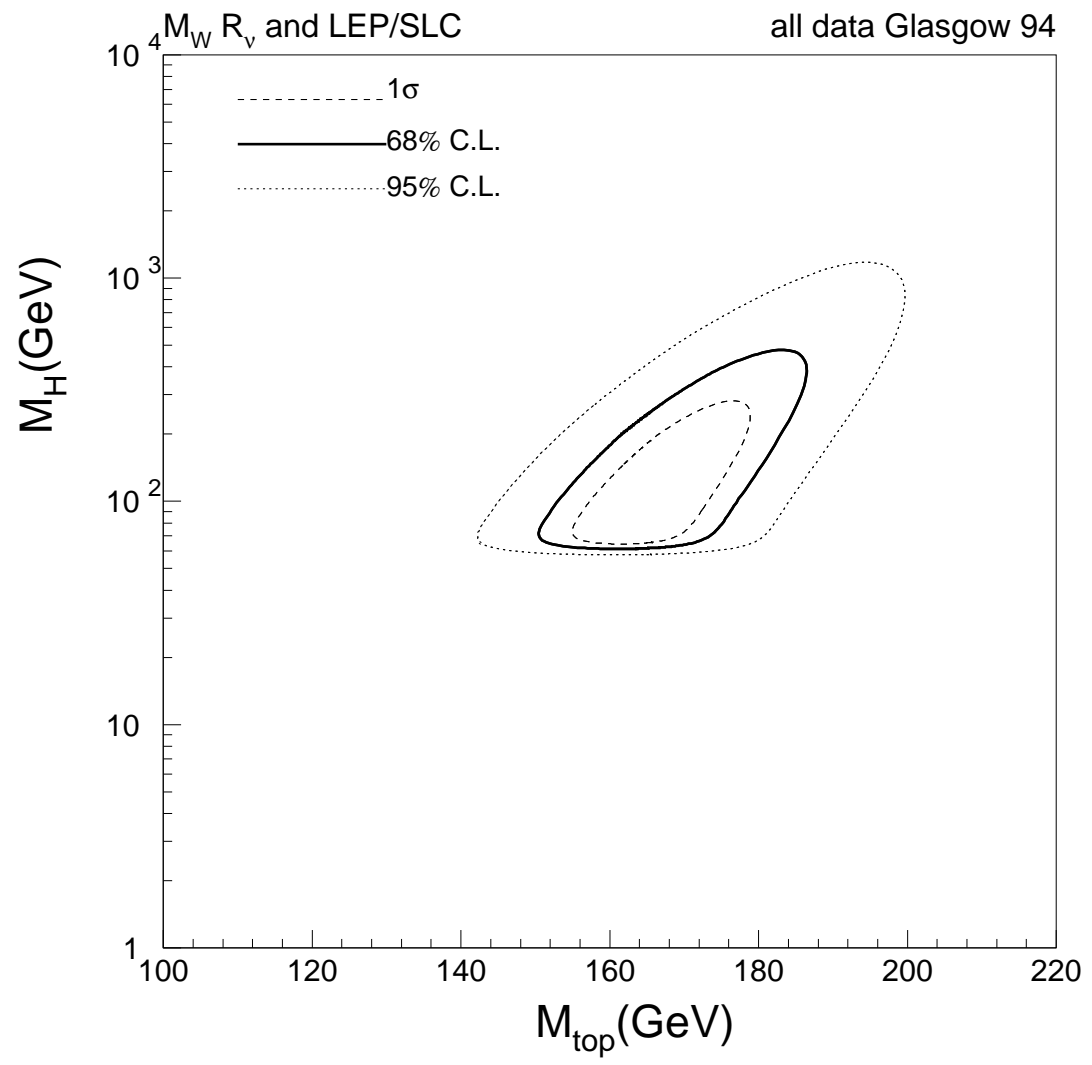

Figure 20: Same as Fig. 19 including also the possible measurement of $\mathrm{M}_{\mathrm{t}}=$ $174 \pm 16 \mathrm{GeV}$. 
Figure 21: A possible scenario for the ultimate precision of electroweak measurements at LEP. SM predictions in the $\left(\Delta \rho, \Delta_{3 \mathrm{Q}}\right)$ plane.

SM predictions. Dash-dotted line: $\mathrm{M}_{\mathrm{t}}$ free, $\mathrm{M}_{\mathrm{H}}=50 \mathrm{GeV}$. Full line: $\mathrm{M}_{\mathrm{t}}$ free, $\mathrm{M}_{\mathrm{H}}=200 \mathrm{GeV}$. Dotted line: $\mathrm{M}_{\mathrm{t}}$ free, $\mathrm{M}_{\mathrm{H}}=1000 \mathrm{GeV}$.

Experimental constraints. Vertical band: $\Delta \Gamma_{\ell}= \pm 0.07 \mathrm{MeV}$ (from $\Delta \Gamma_{\mathrm{Z}}=$ $\pm 2 \mathrm{MeV}$ ). $25^{\circ}$ band: $\Delta \mathrm{M}_{\mathrm{W}}= \pm 30 \mathrm{MeV} .45^{\circ}$ band: $\Delta \sin ^{2} \theta_{\mathrm{w}}^{\text {eff }}= \pm 0.0001$. The $S M$ prediction for $\mathrm{M}_{\mathrm{t}}=165 \pm 5 \mathrm{GeV}$ as expected from a future measurement of the top mass is also indicated. 
the beam energy by the resonant depolarization method. An error of $\pm 2 \mathrm{MeV}$ on $\Gamma_{Z}$ seems reachable. Asymmetry measurements should also improve, resulting in a combined measurement error on $\sin ^{2} \theta_{\mathrm{w}}^{\mathrm{eff}}$ of \pm 0.0003 . More speculatively, longitudinal polarization experiments at $\mathrm{LEP}^{105,106}$ could lead to much improved measurements of $\sin ^{2} \theta_{\mathrm{w}}^{\mathrm{eff}}$, down to \pm 0.0001 . In order to make full use of the future precision measurements of $\sin ^{2} \theta_{\mathrm{w}}^{\text {eff }}$, the present estimate of $\alpha\left(\mathrm{M}_{\mathrm{Z}}^{2}\right)$ should be improved. The present error corresponds to an uncertainty in predicting $\sin ^{2} \theta_{\mathrm{w}}^{\mathrm{eff}}$ from $\mathrm{M}_{\mathrm{Z}}$ of \pm 0.0003 . The improvement of $\alpha\left(\mathrm{M}_{\mathrm{Z}}^{2}\right)$ requires better measurements of $e^{+} e^{-} \rightarrow$ hadrons in the energy region $1-10 \mathrm{GeV}$. It is hoped that the precision on electroweak measurements will be improved to the level of sensitivity that would make its indirect determination through radiative corrections significant. Such an analysis was performed in Ref. 16, stressing the importance of very accurate measurements of $\sin ^{2} \theta_{\mathrm{w}}^{\mathrm{eff}}$ at LEP, see Fig. 21. Clearly, if the Higgs is found, these same measurements may be precise enough to reveal physics beyond the SM.

Of course, the best thing to do is to find the Higgs. The high-energy program of LEP offers a good chance of finding it up to a mass limit of $\mathrm{M}_{\mathrm{H}}=2 \mathrm{E}_{\text {beam }}$ $-100 \mathrm{GeV}$. Here the maximal energy that can be reached by LEP is the critical parameter. If this fails, we will have to wait for the LHC to give us some answer to the mysteries of spontaneous symmetry breaking.

\section{Acknowledgments}

I am grateful to my colleages from the LEP collaborations for giving me access to their preliminary data, especially R. Clare, A. Kounine, P. Renton, M. Roney, D. Schaile, P. Wells, J. Wenninger, and M. Winter. I thank J. E. Augustin and D. Schlatter for careful reading of the manuscript and several precious comments. Useful discussions with many theorist colleagues are gratefully acknowledged. The LEP electroweak working group has performed with great care the averaging of the experimental results. Manel Martinez and Bolek Pietrzyk deserve special credit for several discussions and graphs. I personally owe a lot to my colleagues of ALEPH and of the Polarization Collaboration for enjoyable company and support during these measurements. 


\section{References}

[1] C. Y. Prescott, in Proceedings of the 1980 Int. Symp. on High-Energy Physics with Polarized Beams and Polarized Targets, edited by C. Joseph and J. Soffer (Birkhäuser Verlag, Basel, 1981), p. 34. M. Böhm and W. Hollik, Nucl. Phys. B 204, 45 (1982).

[2] B. W. Lynn and R. G. Stuart, Nucl. Phys. B 253, 84 (1985).

[3] A. Blondel, B. W. Lynn, F. M. Renard, and C. Verzegnassi, Nucl. Phys. B 304, 438 (1988).

[4] M. Veltman, Nucl. Phys. B 123, 89 (1977).

[5] B. W. Lynn, M. E. Peskin, and R. G. Stuart, "Physics at LEP," CERN 86-02, 90 (1986).

[6] D. C. Kennedy and B. W. Lynn, Nucl. Phys. B 322, (1989).

[7] M. Consoli and W. Hollik, in "Physics at LEP1," CERN 89-08, 7 (1989).

[8] M. E. Peskin and T. Takeuchi, Phys. Rev. Lett. 65, 964 (1990); V. A. Novikov, L. B. Okun, and M. I. Visotsky, Nucl. Phys. B 397, 35 (1993).

[9] G. Altarelli and R. Barbieri, Phys. Lett. B 253, 161 (1991); G. Altarelli, R. Barbieri, and S. Jadach, Nucl. Phys. B 369, 3 (1992); Err. Nucl. Phys. B 376 (1992); G. Altarelli et al., Nucl. Phys. B 405, 3 (1993).

[10] A. Blondel, TASI 1991, edited by Ellis, Hill, and Lykken (World Scientific, $1992)$ 283; A. Blondel and C. Verzegnassi, Phys. Lett. B 311, 346 (1993).

[11] The LEP Collaborations, Phys. Lett. B 276, 247 (1992).

[12] B. W. Lynn, SLAC-Pub 5077 (1989); D. Levinthal, F. Bird, R. G. Stuart, and B. W. Lynn, CERN-Th 6094/91.

[13] $\overline{\mathrm{MS}}$ scheme: S. Sarantakos, A. Sirlin, and W. Marciano, Nucl. Phys. B 217, 84 (1983); W. Marciano and A. Sirlin, Phys. Rev. D 29, 75 (1984); A. Sirlin, Phys. Lett. B 232, 123 (1989); A.Sirlin, Nucl. Phys. B 332, 20 (1990).

[14] A. Olshevski, P. Ratoff, and P. Renton, Z Phys. C 60, 643 (1993); P. Gambino and A. Sirlin, Phys. Rev. D 49, R1160 (1994).

[15] H. Burkhardt, F. Jegerlehner, G. Penso, and C. Verzegnassi, Z Phys. C 43 , 497 (1989).

[16] A. Blondel, F. M. Renard, and C. Verzegnassi, Phys. Lett. B 269, 419 (1991).

[17] A. Blondel, A. Djouadi, and C. Verzegnassi, Phys. Lett. B 293, 253 (1992).

[18] ALEPH Coll., Z. Phys. C 53, 1 (1992); Z. Phys. C 60, 71 (1993); Z. Phys C 62, 539 (1994). Updated results for ICHEP 1994, GLS0557, GLS0558.

[19] DELPHI Coll., Nucl. Phys. B 367, 511 (1991); Nucl. Phys. B 417, 3 (1994); Nucl. Phys. B 418, 403 (1994); ICHEP 1994 GLS0301.

[20] L3 Coll., Z. Phys. C 51, 179 (1991); see also Ref. 21. CERN-PPE/94-45 (1994) submitted to Z. Phys C. Updated results for ICHEP 1994, L3 Note 1620 . 
[21] L3 Coll., Phys. Rep. 236 (1993).

[22] OPAL Coll., Z. Phys. C 52, 175 (1991); Z. Phys. C 52, 175 (1991); Z. Phys. C 58, 219 (1993); Z. Phys. C 61, 19 (1994). Updated results for ICHEP 1994, OPAL Physics Note PN-143.

[23] ALEPH Coll., Z. Phys. C 53, 375 (1992).

[24] B. Bloch, EPS conf. (July 1993); ALEPH Note 93-149; see also Ref. 18; D. Bederède et al., SiCAL - A High-precision luminosity calorimeter for ALEPH, to appear in Nucl. Instrum. Methods.

[25] OPAL Coll., preliminary result ICHEP 1994, OPAL PN-142.

[26] M. Merk, talk given at XXIX Rencontres de Moriond, Meribel (1994).

[27] S. Jadach, E. Richter-Was, Z. Was, and B. F. L. Ward, Phys. Lett. B 268, 253 (1991); Comput. Phys. Commun. 70, 305 (1992); S. Jadach and B. F. L. Ward, Phys. Rev. D 40, 3582 (1989).

[28] M. Böhm, A. Denner, and W. Hollik, Nucl. Phys. B 306, 687 (1988); F. A. Berends, R. Kleiss, and W. Hollik, Nucl. Phys. B 304 (1988), p. 712; computer programme BABAMC by R. Kleiss.

[29] S. Jadach, E. Richter-Was, Z. Was, and B. F. L. Ward, Phys. Lett. B 253, 469 (1991); Phys. Lett. B 260, 438 (1991).

[30] W. Beenakker and B. Pietrzyk, Phys. Lett. B 296, 296 (1992); Phys. Lett. B 304, 366 (1993).

[31] B. F. L. Ward, S. Jadach, E. Richter-Was, and Z. Was, talk given at Glasgow 1994, UTHEP-94-0801.

[32] W. Beenakker, F. A. Berends, and S. C. Van der Marck, Nucl. Phys. B 355, 281 (1991).

[33] A. Blondel, Second Workshop on LEP Performance, CERN-SL/92-29 (DI) 339.

[34] A. A. Sokolov and I. M. Ternov, Sov. Phys. Doklady, 8, 1203 (1964).

[35] This effect was first seen in the Orsay ACO storage ring: R. Belbeoch et al., USSR Part. Accel. Conf. 129 (1968). For reviews, see B. W. Montague, Phys. Rep. 113 (1984); A. Blondel CERN-PPE/93-125 (1993); M. Böge, DESY 94-087.

[36] L. Knudsen et al., Phys. Lett. B 270, 97 (1991).

[37] A. A.Zholentz et al., Phys. Lett. B 96, 214 (1980).

[38] A. S. Artamonov et al., Phys. Lett. B 118, 225 (1982).

[39] D. P. Barber et al., Phys. Lett. B 135, 498 (1984).

[40] W. W. McKay et al., Phys. Rev. D 29, 2483 (1984).

[41] L. Arnaudon et al., Phys. Lett. B 284, 431 (1992).

[42] R. Assmann et al., "Accurate determination of the LEP beam energy by resonant depolarization," CERN-SL/94-71(BI), and "Polarization studies at LEP in 1993," CERN-SL/94-08(AP). 
[43] L. Arnaudon et al., "The energy calibration of LEP in 1991," CERNPPE/92-125 (1992) and CERN-SL/92-37(DI); LEP Energy Group, ALEPH, DELPHI, L3, and OPAL Collaborations, L. Arnaudon et al., Phys. Lett. B 307, 187 (1993); L. Arnaudon et al., "The energy calibration of LEP in 1992," CERN SL/93-21 (DI), April 1993.

[44] G. Fischer and A. Hofmann, in the Second Workshop on LEP Performance, edited by J. Poole, CERN-SL/92-29 (DI) 337 (1992).

[45] L. Arnaudon et al., CERN-SL/94-07 (BI) (1994).

[46] Investigation suggested to me by B. Richter and realized by B. Jacobsen, SL-MD Note 62, (1992).

[47] J. Wenninger, CERN-SL/94-14 (BI).

[48] The LEP Collaborations ALEPH, DELPHI, L3, OPAL, and the LEP Electroweak working group, CERN-PPE/93-157; ibid, preliminary averages prepared for the summer conferences, 1994.

[49] F. A. Berends, G. Burgers, W. Hollik, and W. L. van Neerven, Phys. Lett. B 203 (1988) 177; G. Burgers, in Ref. 105, Vol. I, 121; M. Böhm and W. Hollik, in $Z$ Physics at LEP I, CERN 89-08 (1989) Vol. I, 203; F. A. Berends et al., ibid, Vol. I, 89. See also Ref. 50.

[50] D. C. Kennedy et al., Nucl. Phys. B 321, 83 (1989).

[51] M. Martinez et al., Z Phys. C 49, 645 (1991).

[52] D. Bardin et al., Z. Phys. C 44, 493 (1989); Nucl. Phys. B 351, 1 (1991); Phys. Lett. B 255, 290 (1991) and CERN-TH 6443/92 (May 1992); Computer programme ZFITTER, available from D. Y. Bardin.

[53] A. Akhundov, D. Bardin, and T. Riemann, Nucl. Phys. B 276, 1 (1986); W. Beenakker and W. Hollik, Z. Phys. C 40, 141 (1988); B. W. Lynn and R. G. Stuart, Phys. Lett. B 252, 676 (1990); J. Barnabeu, A. Pich, and A. Santamaria, Nucl. Phys. B 363, 326 (1991).

[54] D. Brown, MPI preprint MPI-PhE 93-25 Munich (1993).

[55] ALEPH Coll., Z Phys. C 62, 179 (1994); ALEPH Coll., CERN-PPE/94023 to appear in Nucl. Instrum. Methods; updated results for ICHEP 1994, ALEPH Note 94-123.

[56] DELPHI Coll., Z. Phys. C 56, 201 (1992); updated results for ICHEP 1994, GLS0301b.

[57] L3 Coll., Phys. Lett. B 261, 177 (1991); see also Ref. 21, updated results for ICHEP 1994, L3 Note 1624.

[58] OPAL Coll., Z Phys. C 58, 523 (1993). OPAL Coll., Z Phys. C 60, 199 (1993).

[59] ALEPH Coll., Phys. Lett. B 313, 549 (1993).

[60] DELPHI Coll., Phys. Lett. B 295, 383 (1992).

[61] L3 Coll., Phys. Lett. B 307, 237 (1993).

[62] G. Batignani et al., 1991 IEEE Nuclear Science Symp. (Santa Fe), IEEE Trans. on Nuclear Science V. NS 39(4-5) (1992) Vol. 1, 444; ALEPH Coll., Phys. Lett. B 313, 535 (1993). 
[63] DELPHI Coll., preliminary results for ICHEP 1994, GLS0301b, GLS0229.

[64] OPAL Coll., CERN-PPE/94-106 (1994).

[65] Y. S. Tsai, Phys. Rev. D 4, 2821 (1971); erratum Phys. Rev. D 13, 771 (1976).

[66] G. Goggi, CERN 79-01 (1979) Vol. II, 483; J. E. Augustin, ibid, 499.

[67] ALEPH Coll., Phys. Lett. B 265, 430 (1991); ALEPH Coll., Z. Phys. C 59, 369 (1993); update for ICHEP 1994, GLS0551.

[68] DELPHI Coll., Z. Phys. C 55, 555 (1992); update for ICHEP 1994, GLS0207.

[69] OPAL Coll., Phys. Lett. B 266, 201 (1991); CERN-PPE/94-120 (1994).

[70] A. Rougé, Z. Phys. C 48, 45 (1990).

[71] M. Davier et al., Phys. Lett. B 306, 411 (1993).

[72] ARGUS Coll., Z. Phys. C 58, 61 (1993).

[73] ALEPH Coll., Phys. Lett. B 321, 168 (1994).

[74] ALEPH Coll., Phys. Lett. B 259, 377 (1991).

[75] ALEPH Coll., preliminary results for Moriond 1993, ALEPH-Note 93041, PHYSIC 93-032 (1993); ALEPH-Note 93-042, PHYSIC 93-034 (1993); ALEPH-Note 93-044, PHYSIC 93-036 (1993).

[76] DELPHI Coll., Phys. Lett. B 277, 231 (1992).

[77] OPAL Coll., Phys. Lett. B 294, 436 (1992).

[78] R. D. Field and R. P. Feynman, Nucl. Phys. B 136, 1 (1978).

[79] J. P. Berge et al., Nucl. Phys. B 184, 13 (1981); EMC Coll., Phys. Lett. B 144, 302 (184).

[80] S. Anassontzis et al., Nucl. Instrum. Methods, A 323, 351 (1992).

[81] DELPHI Coll., ICHEP 1994, GLS0232.

[82] DELPHI Coll., Phys. Lett. B 276, 536 (1992); updated results for ICHEP 1994, GLS0301b, GLS0230, GLS0230b.

[83] L3 Coll., Phys. Lett. B 252, 713 (1990); see also Ref. 21; L3 Coll., CRNPPE/94-89 (1994); updated results for ICHEP 1994, L3 Note 1624.

[84] OPAL Coll., Phys. Lett. B 263, 311 (1991); OPAL Coll., Z Phys. C 60, 19 (1993); updated results for ICHEP 1994 OPAL Phys. Note PN118.

[85] G. Altarelli and B. Lampe, Nucl. Phys. B 391, 3 (1993).

[86] ALEPH Coll., CERN-PPE/94-84 (1994).

[87] DELPHI Coll., ICHEP 1994, GLS0230.

[88] OPAL Coll., ICHEP 1994, OPAL Phys. Note PN127.

[89] ALEPH Coll., Z. Phys. C 62, 1 (1994); updated results for ICHEP 1994, GLS0 562 .

[90] DELPHI Coll., Z. Phys. C 59, 533 (1993); updated results for ICHEP 1994, GLS0231. 
[91] OPAL Coll., Z. Phys. C 60, 601 (1993), updated results for ICHEP 1994, OPAL Phys. Note PN125.

[92] D. Abbaneo et al., "A consistent treatment of systematic errors for LEP electroweak heavy flavor analyses," LEPHF/94-01 (1994) unpublished.

[93] T. Junk, talk given at XXIX Rencontres de Moriond, Meribel (1994).

[94] SLD Coll., Phys. Rev. Lett. 73, 25 (1994).

[95] Electroweak libraries: ZFITTER ${ }^{52}$; BHM: G. Burgers, W. Hollik, and M. Martinez; M. Consoli, W. Hollik, and F. Jegerlehner, in CERN 89-08 (1989) Vol. I, 7; G. Burgers, F. Jegerlehner, B. Kniehl, and J. Kühn, ibid, 55. These computer codes have recently been upgraded by including the results of: B. A. Kniehl and A. Sirlin, DESY 92-102; S. Fanchiotti, B. A. Kniehl and A. Sirlin, CERN-TH.6449/92; R. Barbieri et al., Phys. Lett. B 288 (1992) 95; K. G. Chetyrkin and J. H. Kühn, Phys. Lett. B 248 (1990), p. 359; K. G. Chetyrkin, J. H. Kühn, and A. Kwiatkowski, Phys. Lett. B 282, 221 (1992); J. Fleischer, O. V. Tarasov, and F. Jegerlehner, Phys. Lett. B 293, 437 (1992).

[96] R. Barbieri, M. Frigeni, and F. Caravaglios, Phys. Lett. B 279, 169 (1992).

[97] F. Abe et al., (CDF Coll.) "Evidence for top quark production in $\bar{p} p$ collisions at $\sqrt{s}=1.8 \mathrm{TeV}$ " to be published in Phys. Rev D.

[98] A. Blondel et al., Z. Phys. C 45, 361 (1990).

[99] J. V. Allaby et al., (CHARM Coll.): Phys. Lett. B 177, 446 (1986), and Z. Phys. C 36, 611 (1987).

[100] C. G. Arroyo et al., (CCFR Coll.), Phys. Rev. Lett. 72, 3452 (1994).

[101] M. Demarteau et al., "Combining W mass measurements," CDF/PHYS/CDF/PUBLIC/2552, D $\emptyset$ Note 2115 (1994).

[102] J. Alitti et al., (UA2 Coll.) Phys. Lett. B 276, 354 (1992).

[103] F. Abe et al., Phys. Rev. Lett. 65, 2243 (1990); Y. K. Kim, XXIX Rencontres de Moriond, Meribel (1994).

[104] D $\emptyset$ Coll., presented by Q. Zhu at the Ninth Topical Workshop on ProtonAntiproton Collider Physics (1993).

[105] "Polarization at LEP," CERN 88-06, Vols. I, II (1988).

[106] A. Blondel et al., "A test of longitudinal polarization at LEP" CERN/LEPC 94-8, LEPC/P8 (1994). 